\title{
Costruzione di un modello decisionale per valutare la costo/efficacia delle strategie alternative nella terapia della polmonite acquisita in comunità
}

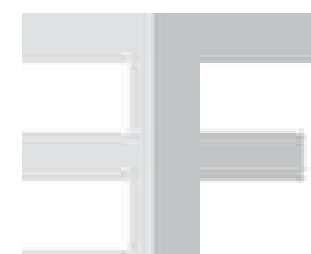

PERCORSI

Mario Eandi $\S$

\begin{abstract}
Aim of this paper was to analyse the cost effectiveness of the main alternative (and complementary) strategies in the disease menagement of the Community-Acquired Pneumonia (CAP): hospital admission vs home-care, antibiotic parental vs oral therapy, switch vs no-switch therapy, and early discarge vs conventional hospitalization.

The cost effectivenessanalysis (CEA) has been performed by implementing a general decision tree model wich describes all the main decisional and change nodes encountered in the clinical course from the firm sign and symptoms of CAP (root) to the final aoutcomes: full recovery or death (terminal nodes).

We assumed the perspectives of three main institutional decision-makers: the society, the italian national healthcare system (NACS), and the hospital. In the perspective of society both the direct (health and non-health) costs and the indirect costs have been included, while in the perspective of the NACS only the health-direct costs were considered. In the perspective of the hospital we considered the overall mean expences sustained for each day of staying in the general and in the intensive care unit. Separately, the antibiotic treatement costs to hospital have been accounted. As effectiveness we considered the percentage of recovery for each class of mortality aqccording to fine.

Most of the probability data used in the model were obtained or derived from the published literature. The cost were valued according to the Italian NACS charges and prices in use during the year 2000.

According to the model structure, the main expenditure factor for the SSN is the hospitalization cost, while the home care is less expensive. The antibiotic parentenal therapy, during hospedalization or home care, is more expensive than the antibiotic oral therapy; but the cost difference between one therapy and the other is clearly lower than the cost difference between the hospitalization and the home care.

The optimum expenditure situation for the SSN, the Society and also for the Hospital coul be obtained by decreasing the days of hospital stay in and by choosing to hospitalize the patients according to the death risk. The sensitivity analysies performed confirmed the robusteness of the results obtained with the model. However the model and its usefulness in decision-making will be definitely confirmed when clinical and epidemiological robust data on CAP in Italy will be available.
\end{abstract}

Farmeconomia e percorsi terapeutici 2001; 2 (1): 37-61

\section{INTRODUZIONE}

La polmonite acquisita in comunità (CAP) è una patologia infettiva che riveste una gran importanza clinica e sociale per la sua incidenza annuale, per l'elevata mortalità e per il carico economico sul Sistema Sanitario Nazionale (SSN) e sulla società (1-7).

Obiettivo di questo lavoro è analizzare la costo-efficacia delle principali strategie alternative e/o complementari che si possono adottare nella gestione del paziente affetto da CAP.

Per analizzare questo problema abbiamo costruito un modello decisionale utilizzando come strumento l'albero delle decisioni.

L'analisi farmacoeconomica utilizza tre principali strategie per generare informazioni sui costi e benefici dei programmi terapeutici: 1) at- tuare una valutazione economica integrata con trias clinici controllati e comparativi; 2) attuare una valutazione dei costi e delle conseguenze dei trattamenti come si realizzano nella pratica clinica quotidiana; 3) simulare i percorsi diagnostici e terapeutici relativi alla gestione del problema sanitario indagato utilizzando modelli decisionali come l'albero delle decisioni o le catene di Markov.

In questo lavoro abbiamo scelto la terza via, quella di utilizzare un modello decisionale, per analizzare il problema della gestione del paziente affetto da polmonite acquisita in comunità (CAP).

Infatti non avevamo a disposizione dati originali ottenuti da trials clinici controllati o da ricerche cliniche osservazionali, ma solo informazioni ricavate dalla letteratura e prese in ras-
§ Dipartimento di Anatomia, Farmacologia e Medicina Legale, Sezione di Farmacologia, Università di Torino. 


\section{Figura 1}

Radice e primi nodi dell'albero decisionale costruito per analizzare la costoefficacia delle strategie alternative adottabili nella terapia della polmonite acquisita in comunità.

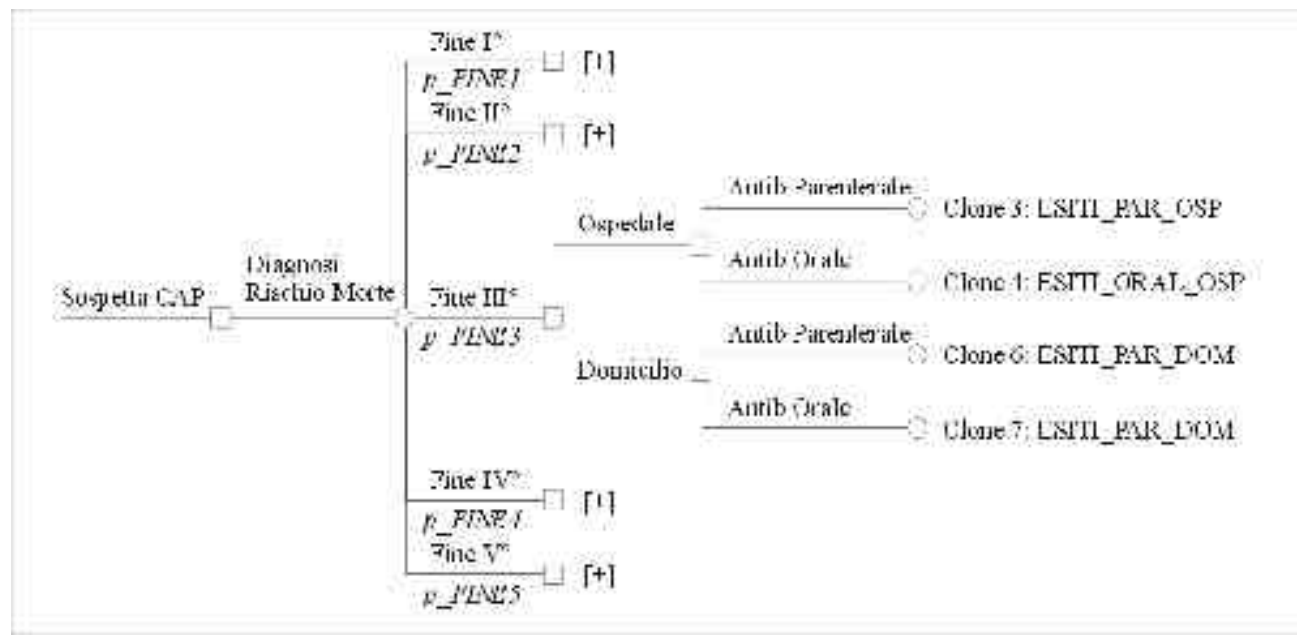

segna nel lavoro precedentemente pubblicato su questa rivista (1).

\section{COSTRUZIONE DELL'ALBERO DELLE DECISIONI}

L'albero delle decisioni è uno strumento molto utile per assumere decisioni ragionate e ponderate (8). Si tratta di un approccio metodologico che consente di rappresentare in modo organico il problema sul quale si deve decidere, le alternative possibili e praticabili, $\mathrm{i}$ nodi decisionali e le probabilità delle relative conseguenze, $i$ costi ed $i$ benefici correlabili a ciascun percorso decisionale.

Questo approccio modellistico è in grado di supplire alla mancanza di dati ricavati direttamente dalla esperienza clinica e di prefigurare scenari anche molto complessi difficilmente indagabili con ricerche cliniche sul campo (9).

L'analisi farmacoeconomica mediante simulazione di modelli decisionali si sviluppa attraverso varie tappe fondamentali: 1) identificazione e definizione del problema decisionale; 2) descrizione del problema decisionale nella sua dimensione temporale; 3) identificazione e quantificazione delle informazioni necessarie; 4) valutazione e scelta di un percorso in base a criteri predefiniti (es. indice costo/efficacia); 5) analisi di sensibilità per stabilire la dipendenza dei risultati dalla variazione di parametri incerti o mal definibili.

I punti 1-3 sono stati affrontati e descritti nel precedente lavoro (1) ed in questa sede vengono solo parzialmente integrati per quanto riguarda aspetti tecnici inerenti alla valutazione modellistica.

\section{PROSPETTIVE DELL'ANALISI}

L'analisi è stata effettuata assumendo le prospettive di tre differenti decisori istituzionali: la Società, il Sistema Sanitario Nazionale (SSN) e l'Ospedale.

\section{A. La struttura}

L'albero delle decisioni, che abbiamo appositamente progettato, rappresenta i nodi decisionali fondamentali che si affrontano nella gestione della CAP: ricoverare il paziente o trattarlo a domicilio, utilizzare antibiotici per via parenterale o per via orale, passare dalla terapia parenterale a quella orale (switch therapy) quando le condizioni cliniche del paziente si stabilizzano e migliorano oppure continuare con l'antibiotico parenterale, dimettere precocemente il paziente oppure trattenerlo in ospedale fino al termine del ciclo terapeutico.

L'albero delle decisioni che abbiamo costruito rappresenta il percorso completo di un paziente dall'insorgenza dei primi sintomi che fanno porre il sospetto diagnostico di CAP fino alla risoluzione definitiva del problema, dicotomizzata come guarigione o come morte del paziente.

La struttura dell'albero è abbastanza complessa e di grandi dimensioni tanto che non è graficamente rappresentabile in una sola pagina: qui viene presentata scomposta nelle figure 1-3.

La figura 1 rappresenta la radice ed i primi nodi dell'albero. La radice indica che il percorso inizia con la diagnosi presuntiva di polmonite. L'approfondimento diagnostico porta non solo a porre diagnosi definitiva ma anche a stimare il rischio di morte del paziente e quindi a classificarlo in una delle 5 classi di rischio definite dall'algoritmo proposto e validato da Fine (10).

Il modello decisionale stratifica, dunque, la pi caratterizzati da una differente gravità delle condizioni cliniche e da un diverso livello di rischio di morte. La gravità della polmonite ed il rischio di morte condizionano, o dovrebbero popolazione dei pazienti affetti da CAP in 5 grup- 


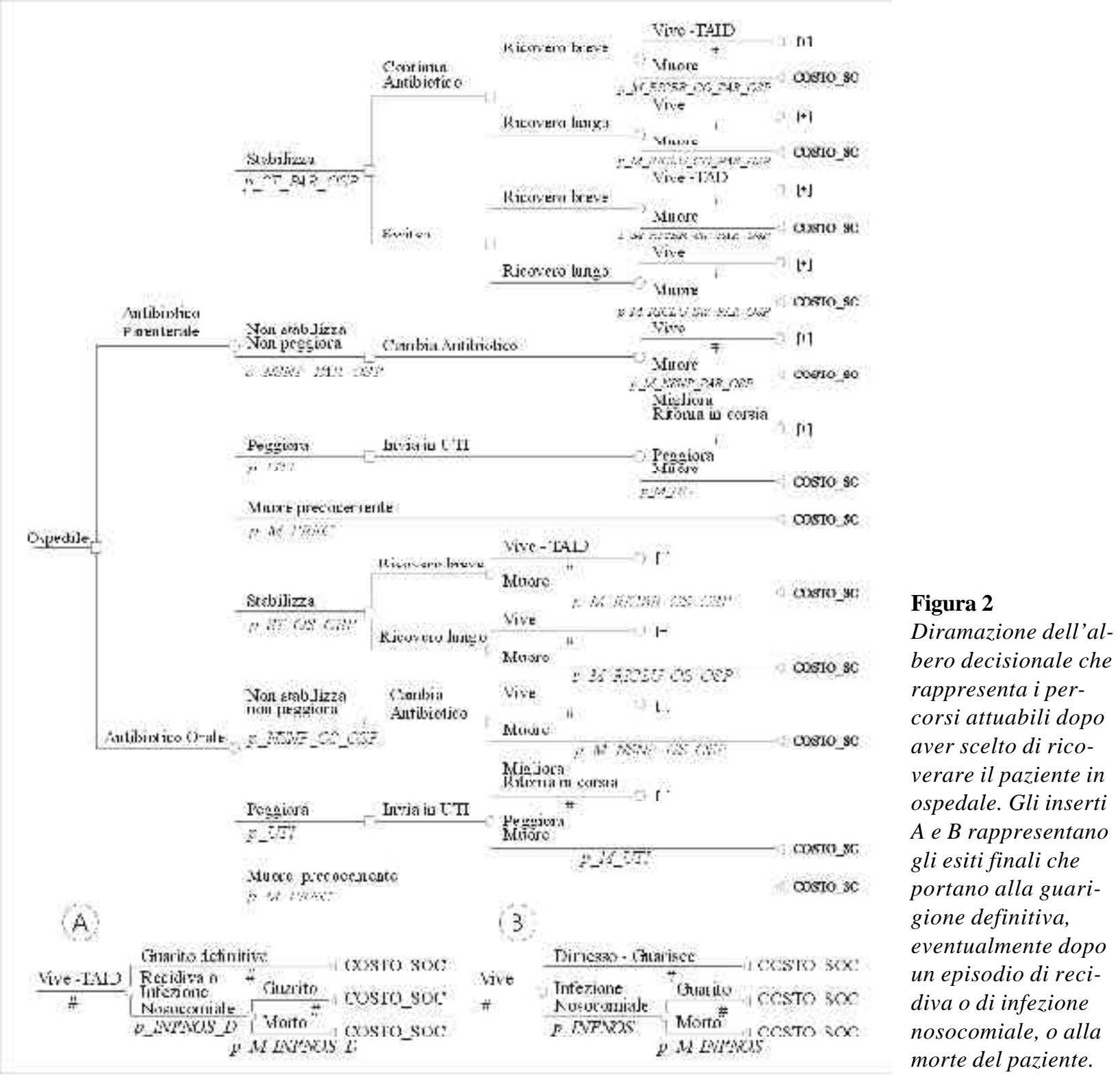

condizionare, fortemente le decisioni di ricoverare il paziente in ospedale e di trattarlo con antibiotici per via parenterale e sono quindi tra i fattori più importanti nel determinare il tipo e la quantità di risorse impiegate.

La figura 2 rappresenta i percorsi attuabili dopo aver scelto di ricoverare il paziente, mentre la figura 3 i percorsi attuabili dopo aver deciso di trattare il paziente a domicilio. In entrambi i casi sono previste due alternative nell'impostazione della terapia antibiotica: attuare una terapia aggressiva per via parenterale oppure una terapia meno aggressiva per via orale.

Nei primi giorni dopo l'inizio della terapia antibiotica il paziente può stabilizzarsi ed iniziare a migliorare, oppure può non rispondere in modo sufficiente al trattamento e richiedere un cambiamento di antibiotico, può peggiorare tanto da richiedere il trasferimento in un'unità di terapia intensiva (UTI) se già è ricoverato, $o$ il ricovero se viene trattato a domicilio, ed infine può peggiorare rapidamente e morire precocemente senza che si possa far nulla di utile. Queste quattro evenienze sono rappresentate come diramazioni del nodo probabilistico che indica le conseguenze immediate della scelta iniziale dell'antibiotico.

Per i soggetti che, trattati con antibiotici iniettivi, stabilizzano e migliorano si pongono altre scelte strategiche: semplificare la terapia e passare alla via orale (switch therapy) oppure continuare con l' antibiotico iniettivo e, nel caso di soggetti ricoverati, dimettere precocemente il paziente stabilizzato oppure trattenerlo fino al termine della cura. Per i soggetti trattati inizialmente a domicilio che non rispondono alla terapia e tendono a peggiorare si impone la decisione di provvedere con un ricovero.

I soggetti che vengono ricoverati per polmonite comunitaria vanno incontro ad un rischio ulteriore, quello di essere colpiti da infe- 


\section{Figura 3}

Diramazione dell'albero decisionale che rappresenta i percorsi attuabili dopo aver scelto di trattare il paziente a domicilio. Gli inserti A e B rappresentano gli esiti finali che portano alla guarigione definitiva, eventualmente dopo un ricovero ospedaliero per insuccesso o recidiva o infezione

nosocomiale, o alla morte del paziente.

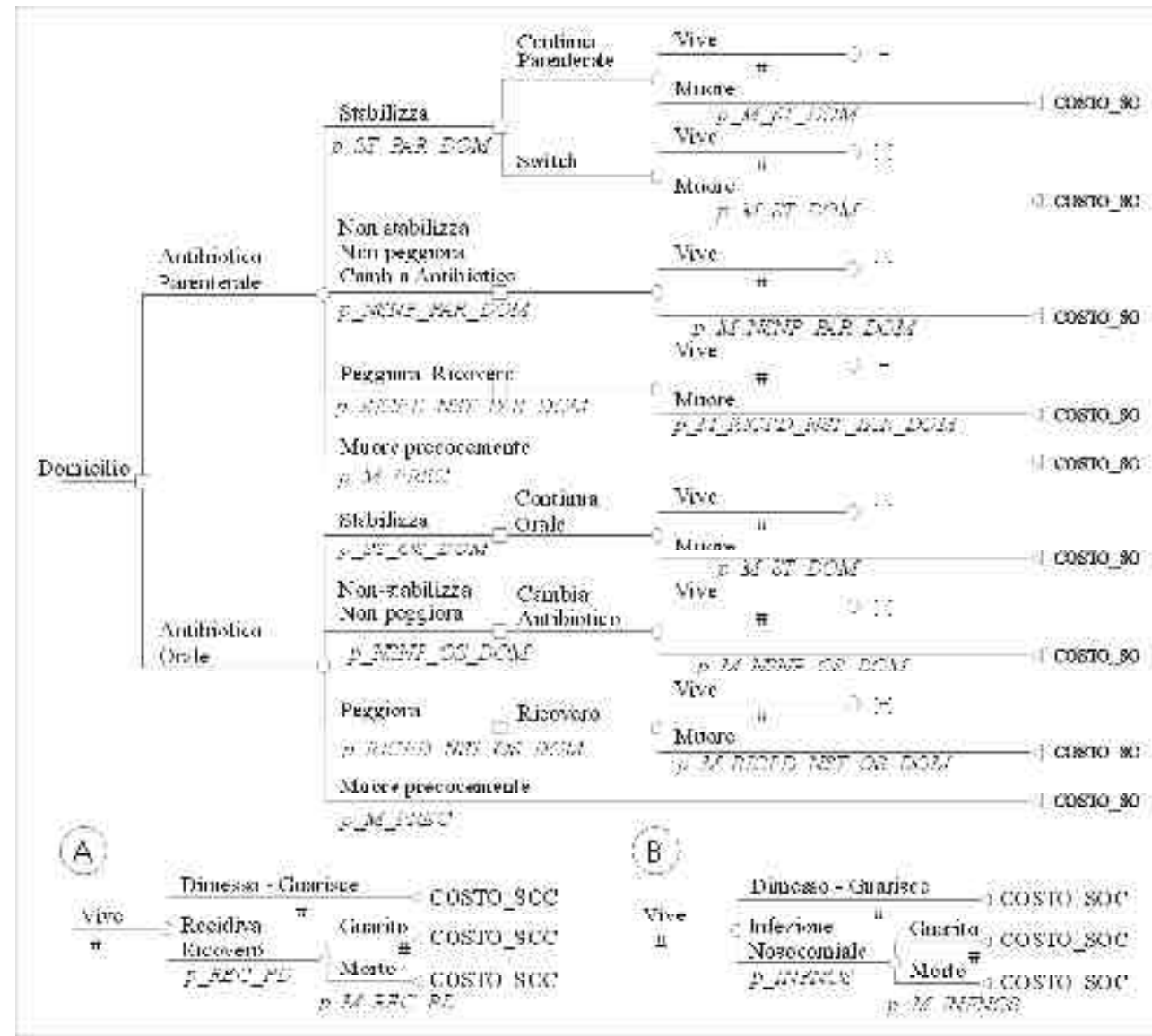

zione nosocomiale, la cui probabilità dipende in qualche misura dalla durata della degenza. Il rischio è minore (ma non nullo) per quei pazienti che vengono dimessi precocemente. L'infezione nosocomiale può manifestarsi durante il ricovero oppure anche a domicilio entro qualche giorno dalla dimissione. In questo caso, se l'infezione ha localizzazione polmonare, può non essere facilmente distinguibile da una recidiva.

L'albero decisionale rappresenta la possibilità che insorga un' infezione nosocomiale nei pazienti che sono o sono stati ricoverati. Inoltre rappresenta anche la recidiva. Infezione e recidiva comportano un nuovo ricovero o il prolungamento della degenza.

Come esiti finali abbiamo considerato solo la guarigione e la morte del paziente, mentre, per semplificare il modello, non abbiamo rappresentato i casi che vanno incontro a sequele prolungate e ad eventuali cronicizzazioni. D'altra parte, mentre abbiamo potuto utilizzare dati robusti di mortalità pubblicati in letteratura $(7$, 10-11), non abbiamo trovato dati precisi e significativi circa la frequenza di sequele prolungate e di cronicizzazione.

\section{B. Le probabilità dei vari percorsi}

In un albero decisionale ogni soggetto, partendo dalla radice, attua un percorso unico fino ad uno dei rami terminali. Le diramazioni che emanano da un nodo decisionale (indicato convenzionalmente con un quadratino) dipendono dal decisore e, quindi, non sono soggette a valutazione probabilistica. Le conseguenze di una scelta sono invece interpretabili in chiave probabilistica e vengono rappresentate come diramazioni da un nodo probabilistico (indicato convenzionalmente con un cerchio). Ad ogni nodo probabilistico il paziente incontra diverse diramazioni caratterizzate da differenti livelli di probabilità di realizzazione.

Un nodo decisionale può essere trasformato in nodo probabilistico se alla decisione si sostituisce la frequenza osservata (ossia la probabilità) con cui le diverse decisioni vengono attuate in un dato sistema sanitario: ad esempio la decisione di ricoverare o meno il paziente può essere trasformata in probabilità che il paziente venga ricoverato quando si trova in un determinato contesto socio-assistenziale. In alcune analisi che abbiamo effettuato per verificare quali siano i costi medi di trattamento della CAP in condizioni naturalistiche, abbiamo sostituito i nodi decisionali con nodi probabilistici, imponendo come probabilità le frequenze normalizzate con cui si realizzano gli eventi alternativi: ricovero versus non ricovero, antibiotico parenterale versus orale, switch therapy versus continuazione dell'antibiotico parenterale, dimissione precoce versus ricovero convenzionale. La frequenza di ricoveri per 
classe di rischio è stata desunta dalla letteratura e rispecchia la condizione socio-economica degli Stati Uniti. Le frequenze relative al verificarsi di terapie parenterali, switch therapy e dimissione precoci sono state ipotizzate sulla base di dati indicativi ma non verificati da alcuna indagine affidabile.

Nella tabella 1 sono riportate le probabilità imposte al modello, come desunte direttamente dalla letteratura o dopo averle opportunamente trasformate in base alla struttura dell'albero $\mathrm{o}$ ad ipotesi aggiuntive (1).

Nella tabella 2 sono riportate le probabilità che abbiamo ipotizzato valide in un assetto socio-economico immaginario ma verosimile.

La probabilità di incorrere in una infezione nosocomiale secondaria è stata modellizzata mediante una funzione distributiva dipendente dalla durata del ricovero: è stato imposto che il rischio di infezione fosse nullo per degenze inferiori a 2 giorni e che il rischio aumentasse in modo esponenziale fino ad un massimo dell' $8 \%$ per degenze via via più lunghe.

\section{Struttura dei costi}

Sono stati considerati i costi diretti sanitari, i costi diretti non sanitari ed i costi indiretti.

Nella prospettiva del SSN sono stati valorizzati i costi diretti sanitari, mentre nella prospettiva della Società, oltre ai costi diretti sanitari, sono stati valorizzati anche i costi diretti non sanitari, sostenuti prevalentemente dal paziente e dai suoi familiari, ed i costi indiretti.

Nella tabella 3 è riportata la struttura dei costi adottata nel presente modello nella prospettiva della Società e del SSN.

Costituiscono il costo diretto sanitario la componente "ricovero" e la componente "assistenza a domicilio". Nel caso del trattamento antibiotico iniettivo effettuato a domicilio (TAID) abbiamo ipotizzato l'intervento del SSN sotto forma di un programma di assistenza domiciliare integrata (ADI) che prevede la visita giornaliera di un infermiere e la visita periodica del medico, con cadenze che dipendono dall'andamento clinico del paziente.

Secondo l'attuale sistema di remunerazione delle prestazioni sanitarie, i costi di un ricovero ospedaliero pesano sul SSN per il valore della

\begin{tabular}{|lccccc}
\hline VARIABILE & FINE I $^{\circ}$ & FINE II $^{\circ}$ & FINE III $^{\circ}$ & FINE IV $^{\circ}$ & FINE V $^{\circ}$ \\
\hline $\begin{array}{l}\text { Probabilità di appartenere } \\
\text { alla classe di rischio }\end{array}$ & 0,337 & 0,209 & 0,143 & 0,212 & 0,099 \\
\hline $\begin{array}{l}\text { Probabilità di morire } \\
\text { entro 30 giorni }\end{array}$ & 0,005 & 0,009 & 0,012 & 0,09 & 0,271 \\
\hline \hline $\begin{array}{l}\text { Probabilità di ricovero } \\
\text { ospedaliero immediato }\end{array}$ & 0,24 & 0,49 & 0,78 & 0,92 & 0,996 \\
\hline $\begin{array}{l}\text { Probabilità di stabilizzare le } \\
\text { condizioni cliniche entro pochi } \\
\text { giorni con il primo antibiotico }\end{array}$ & 0,9 & 0,85 & 0,80 & 0,70 & 0,35 \\
\hline $\begin{array}{l}\text { Probabilità di non stabilizzare } \\
\text { e di dover cambiare antibiotico } \\
\text { durante il ricovero }\end{array}$ & 0,05675 & 0,10655 & 0,1404 & 0,1815 & 0,46345 \\
\hline $\begin{array}{l}\text { Probabilità di dover } \\
\text { essere trasferito in UTI }\end{array}$ & 0,043 & 0,043 & 0,059 & 0,114 & 0,173 \\
\hline $\begin{array}{l}\text { Probabilità di morte } \\
\text { precoce dopo inizio terapia }\end{array}$ & 0,00025 & 0,00045 & 0,0006 & 0,0045 & 0,01355 \\
\hline
\end{tabular}

Probabilità di morire durante il ricovero in UTI

Probabilità di peggioramento con ricovero ospedaliero dopo iniziale terapia a domicilio

Probabilità di non stabilizzare e di cambiare antibiotico durante la terapia a domicilio

$\begin{array}{lllll}0,08 & 0,12 & 0,16 & 0,4 & 0,6\end{array}$

$\begin{array}{lllll}0,051 & 0,082 & 0,167 & 0,2 & 0,6\end{array}$

Tabella 1

Probabilità imposte al modello, come desunte dalla letteratura o modificate in base alla struttura del modello elo ad ipotesi aggiuntive. Le probabilità sono ripartite per classe di rischio di morte secondo Fine. 
Tabella 2

Probabilità imposte al modello in base ad ipotesi ragionevoli ma non comprovate da dati oggettivi. Sono indicati $i$ valori base e quelli minimi e massimi utilizzati in alcune analisi di sensibilità.

\begin{tabular}{|c|c|c|c|}
\hline VARIABILE & $\begin{array}{l}\text { Valore } \\
\text { base }\end{array}$ & Min & Max \\
\hline Probabilità di terapia antibiotica parenterale in ospedale & 0,8 & 0,5 & 0,95 \\
\hline Probabilità di terapia antibiotica parenterale a domicilio & 0,5 & 0,1 & 0,7 \\
\hline Probabilità di switch therapy in ospedale & 0,4 & 0,2 & 0,6 \\
\hline Probabilità di switch therapy a domicilio & 0,4 & 0,2 & 0,6 \\
\hline $\begin{array}{l}\text { Probabilità di ricovero breve in soggetti con antibiotico } \\
\text { parenterale }\end{array}$ & 0,2 & 0,1 & 0,4 \\
\hline $\begin{array}{l}\text { Probabilità di ricovero breve in soggetti con antibiotico } \\
\text { orale }\end{array}$ & 0,4 & 0,2 & 0,6 \\
\hline $\begin{array}{l}\text { Probabilità di ricovero breve in soggetti dopo switch } \\
\text { therapy }\end{array}$ & 0,6 & 0,4 & 0,8 \\
\hline $\begin{array}{l}\text { Probabilità di recidiva e } 2^{\circ} \text { ricovero dopo dimissione } \\
\text { dall'ospedale }\end{array}$ & 0,02 & 0,01 & 0,04 \\
\hline Probabilità di morte dopo recidiva & 0,1 & 0,02 & 0,2 \\
\hline Probabilità di morte per infezione secondaria nosocomiale & 0,1 & 0,02 & 0,2 \\
\hline Frazione di morti precoci sul totale di morti & 0,05 & 0,01 & 0,1 \\
\hline Frazioni di morti in soggetti che hanno stabilizzato & 0,01 & 0,001 & 0,02 \\
\hline Frazione di morti in UTI sul totale & 0,90 & 0,80 & 0,95 \\
\hline $\begin{array}{l}\text { Frazione di morti in soggetti che devono cambiare } \\
\text { antibiotico }\end{array}$ & 0,04 & 0,01 & 0,1 \\
\hline
\end{tabular}

tariffa DRG liquidata. Tuttavia, il costo reale di l'ospedale per curare i pazienti affetti da polproduzione del servizio ospedaliero può esse- monite, abbiamo tentato di ricostruire il costo re significativamente diverso dal valore della di un ricovero imponendo un valore forfetario tariffa DRG incassata: questo risultato è proba- medio ad ogni giornata di degenza consumata bile soprattutto con alcuni tipi di pazienti che, a in corsia o in Unità di Terapia Intensiva (UTI). causa delle loro condizioni cliniche, consuma- Il costo forfetario di una giornata di degenza no una quantità di risorse inferiore o superiore comprende anche la quota relativa alle terapie al valore medio previsto dalla tariffa. Nella pro- antibiotiche. A parte abbiamo stimato i costi spettiva del decisore che gestisce l'ospedale è sostenuti dall'ospedale per effettuare la teraimportante poter confrontare il valore della ta- pia antibiotica. Il confronto tra il costo del ricoriffa DRG con i costi reali sostenuti per diverse vero ed il costo della terapia antibiotica è un tipologie di pazienti. Da tale confronto il respon- parametro utile per verificare se e dove sia consabile della gestione dell'ospedale può trarre indicazioni per migliorare l'efficienza del servizio. Tale operazione è utile anche per la società e per il SSN. Infatti, nel caso in cui il costo di gestione ospedaliera superasse sistematicamente il valore del DRG, il disavanzo finirebbe per gravare sulla società, costretta a ripianare il deficit con un aumento delle tasse o della quota di compartecipazione. Nel caso in cui, invece, il valore del DRG liquidato fosse sistematicamente superiore al costo di gestione ospedaliera, il SSN avrebbe interesse a ritoccare verso il basso la tariffa. Non avendo a disposizione dati analitici sui costi sostenuti dal- veniente risparmiare sulla spesa farmaceutica.

Nella tabella 4 è riportata la struttura dei costi di produzione del servizio di assistenza ospedaliera e quella dei costi della terapia antibiotica ospedaliera, utilizzate nel modello.

Per stimare i costi abbiamo analizzato i possibili consumi di risorse correlabili ai diversi percorsi terapeutici e quindi abbiamo valorizzato le risorse consumate in unità monetarie (Lire) fissando il periodo all'anno 2000.

Nella maggior parte dei casi sono state stimate le risorse consumate giornalmente e, separatamente, la durata in giorni di ciascun 


\begin{tabular}{|lcc}
\hline TIPO DI COSTI & SSN & SOCIETA' \\
\hline DIRETTI SANITARI & SI & SI \\
\hline 1. Ricoveri ospedalieri (DRGs) & SI & SI* \\
\hline 2. Assistenza domiciliare & SI & SI \\
\hline $\begin{array}{l}\text { a. Terapia antibiotica } \\
\text { i. Antibiotici della fase iniziale } \\
\text { ii. Antibiotici della fase finale } \\
\text { iii. Antibiotici della fase post-ricovero }\end{array}$ & SI & SI \\
\hline
\end{tabular}

\begin{tabular}{lll}
\hline \hline b. Altri farmaci & SI & SI \\
\hline c. Visite mediche & SI & SI \\
\hline d. Assistenza infermieristica & SI & SI \\
\hline e. Esami diagnostici & SI & SI
\end{tabular}

i. Radiologici

ii. Microbiologici

iii. Chimico-clinici

\begin{tabular}{|lll}
\hline DIRETTI NON SANITARI & NO & SI \\
\hline 1. Trasporto in ambulanza & NO & SI \\
\hline \hline
\end{tabular}

Tabella 3

Struttura dei costi adottata nel model-

\begin{tabular}{lll}
\hline 2. Viaggi da casa ad ospedale & NO & SI
\end{tabular}

\begin{tabular}{|c|c|c|}
\hline 3. Assistenza integrativa familiari & $\mathrm{NO}$ & SI \\
\hline
\end{tabular}

\begin{tabular}{lll} 
4. Piccole spese aggiuntive & NO & SI \\
\hline
\end{tabular}

\begin{tabular}{lll}
\hline INDIRETTI & NO & SI \\
\hline 1. Perdita di produttività & NO & SI
\end{tabular}
lo di analisi costoefficacia della terapia della CAP secondo la prospettiva del SSN e della Società.

* Sulla Società gravano i costi reali di produzione del servizio ospedaliero nel caso in cui questi siano superiori al valore del DRG.

\section{COSTO GLOBALE DEL RICOVERO}

2. Costo ricovero in corsia per paziente non complicato

a. Costo giornaliero di assistenza

b. $\mathrm{N}^{\circ}$ giorni in corsia

3. Costo ricovero in UTI

a. Costo giornaliero di ricovero in UTI

b. $\mathrm{N}^{\circ}$ giorni in UTI

4. Costo ricovero paziente complicato dopo terapia domiciliare

a. Costo giornaliero di ricovero

b. $\mathrm{N}^{\circ}$ giorni di ricovero

5. Costo ricovero per recidiva o infezione nosocomiale

a. Costo giornaliero di ricovero

b. $\mathrm{N}^{\circ}$ giorni di ricovero

\section{COSTO TERAPIA ANTIBIOTICA IN OSPEDALE}

1. Antibiotico della fase iniziale

a. Costo giornaliero terapia iniziale

b. $\mathrm{N}^{\circ}$ giorni terapia della fase iniziale

2. Antibiotico della fase finale

a. Costo giornaliero della terapia antibiotica finale

b. $\mathrm{N}^{\circ}$ giorni terapia della fase finale

\section{Antibiotico in UTI}

a. Costo giornaliero della terapia in UTI

b. $\mathrm{N}^{\circ}$ giorni in UTI

Tabella 4

Struttura dei costi di produzione del servizio ospedaliero adottata nel modello di analisi costoefficacia della terapia della CAP secondo la prospettiva del decisore Ospedale. 


\begin{tabular}{|c|c|c|c|}
\hline $\begin{array}{l}\text { COSTI A CARICO DEL SISTEMA SANITARIO } \\
\text { NAZIONALE E/O DELLA SOCIETA' }\end{array}$ & $\begin{array}{l}\text { Valore } \\
\text { base }\end{array}$ & Min & Max \\
\hline \multicolumn{4}{|c|}{$\begin{array}{l}\text { Costo giornaliero a carico del SSN per acquisto antibiotico e materiali in caso di } \\
\text { terapia domiciliare }\end{array}$} \\
\hline Antibiotico orale per soggetti in classe Fine 1-3 & 12.500 & 6.700 & 22.000 \\
\hline Antibiotico orale per soggetti in classe Fine 4-5 & 26.000 & 19.000 & 36.000 \\
\hline Antibiotico orale scelto dopo insuccesso iniziale & 26.000 & 19.000 & 36.000 \\
\hline Antibiotico orale scelto per la switch therapy & 12.500 & 6.700 & 22.000 \\
\hline Antibiotico parenterale per soggetti in classe Fine 1-3 & 38.000 & 16.000 & 68.000 \\
\hline Antibiotico parenterale per soggetti in classe Fine 4-5 & 71.000 & 20.000 & 200.000 \\
\hline Antibiotico parenterale scelto dopo insuccesso iniziale & 71.000 & 20.000 & 200.000 \\
\hline \multicolumn{4}{|l|}{ Altri costi diretti sanitari } \\
\hline Altri farmaci a domicilio, esclusi gli antibiotici & 2.000 & 500 & 5.000 \\
\hline Esami di laboratorio per episodio & 150.000 & 100.000 & 250.000 \\
\hline Esami microbiologici per episodio & 100.000 & 80.000 & 150.000 \\
\hline Esami radiologici per episodio & 60.000 & 30.000 & 90.000 \\
\hline Assistenza infermieristica a domicilio (per accesso) & 20.000 & 15.000 & 30.000 \\
\hline Visita medica a domicilio & 50.000 & 40.000 & 60.000 \\
\hline \multicolumn{4}{|l|}{ Costi diretti non sanitari } \\
\hline Assistenza integrativa dei familiari o volontari & 30.000 & 15.000 & 50.000 \\
\hline Piccole spese aggiuntive & 2.000 & 500 & 5.000 \\
\hline Viaggi e trasferimenti & 3.000 & 1.500 & 5.000 \\
\hline Trasporto ambulanza & 50.000 & 40.000 & 80.000 \\
\hline \multicolumn{4}{|l|}{ Costi indiretti } \\
\hline Perdita di produttività & 100.822 & 80.000 & 150.000 \\
\hline \multicolumn{4}{|l|}{ Tariffe DRG applicate per i ricoveri pagati dal SSN } \\
\hline Tariffa DRG89: polmonite dell'adulto con complicazioni & 6.517 .000 & 6.000 .000 & 8.000 .000 \\
\hline $\begin{array}{l}\text { Tariffa DRG90 : polmonite nell'adulto senza } \\
\text { complicazioni }\end{array}$ & 4.349 .000 & 00.000 & 5.500 .000 \\
\hline $\begin{array}{l}\text { Tariffa DRG applicata nel modello in caso di secondo } \\
\text { ricovero }\end{array}$ & 5.000 .000 & 4.500 .000 & 7.000 .000 \\
\hline COSTI A CARICO DELL'OSPEDALE & $\begin{array}{l}\text { Valore } \\
\text { base }\end{array}$ & Min & Max \\
\hline
\end{tabular}

Costo giornaliero acquisto antibiotico, materiali e lavoro per trattamento antibiotico in ospedale

\begin{tabular}{|lrrr}
\hline Antibiotico orale per soggetti in classe Fine 1-3 & 10.000 & 7.000 & 14.000 \\
\hline Antibiotico orale per soggetti in classe Fine 4-5 & 21.500 & 16.000 & 28.000 \\
\hline Antibiotico orale scelto dopo insuccesso iniziale & 21.500 & 16.000 & 28.000 \\
\hline Antibiotico orale scelto per la switch therapy & 10.000 & 7.000 & 14.000 \\
\hline Antibiotico parenterale per soggetti in classe Fine 1-3 & 51.500 & 20.000 & 86.000 \\
\hline Antibiotico parenterale per soggetti in classe Fine 4-5 & 93.000 & 29.000 & 231.000 \\
\hline Antibiotico parenterale scelto dopo insuccesso iniziale & 93.000 & 29.000 & 231.000 \\
\hline Antibiotico per pazienti in UTI & 160.000 & 80.000 & 250.000 \\
\hline
\end{tabular}

Costo giornaliero medio forfetario per produzione assistenza ospedaliera

Tabella 5

Valori monetari applicati alle principali variabili di costo del modello decisionale.

\begin{tabular}{llll}
\hline Assistenza in corsia per ricoverati con recidiva & 650.000 & 500.000 & 800.000 \\
\hline Assistenza in corsia per primo ricovero & 550.000 & 400.000 & 800.000 \\
\hline \hline $\begin{array}{l}\text { Assistenza in corsia per paziente ricoverato dopo } \\
\text { iniziale terapia domiciliare }\end{array}$ & 650.000 & 500.000 & 800.000 \\
\hline Assistenza in UTI & 2.500 .000 & 2.000 .000 & 3.000 .000
\end{tabular}




\begin{tabular}{|c|c|c|c|c|c|c|c|c|}
\hline \multicolumn{2}{|c|}{ Antibiotico Orale } & \multicolumn{3}{|c|}{$\begin{array}{l}\text { Soggetti trattati a domicilio } \\
\text { Costo Giornaliero per il SSN }\end{array}$} & \multicolumn{3}{|c|}{$\begin{array}{c}\text { Soggetti ricoverati } \\
\text { Costo Giornaliero per l'Ospedale }\end{array}$} & \\
\hline Antibiotico & $\begin{array}{l}\text { Posologia } \\
\text { giornaliera }\end{array}$ & $\begin{array}{r}\text { Prezzo } \\
\text { (Lire) }\end{array}$ & $\begin{array}{l}\text { Costo } \\
\text { Acquisto* }\end{array}$ & $\begin{array}{c}\text { Correzione } \\
\text { Spreco }^{\circ}\end{array}$ & $\begin{array}{l}\text { Costo } \\
\text { acquisto }\end{array}$ & $\begin{array}{c}\text { Costo } \\
\text { Lavoro§ }\end{array}$ & $\begin{array}{l}\text { Totale } \\
\text { Ospedale }\end{array}$ & \\
\hline \multicolumn{8}{|c|}{ AMOXICILLINA/CLAV } & \\
\hline $1 \mathrm{~g} \times 2$ os & & 6.000 & 5.820 & 6.693 & 3.000 & 4.000 & 7.000 & \\
\hline $1 \mathrm{~g} \times 3$ os & & 9.000 & 8.730 & 10.040 & 4.500 & 6.000 & 10.500 & \\
\hline \multicolumn{8}{|c|}{ CEFUROXIM AXETIL } & \\
\hline $500 \mathrm{mg} \times 2$ & & 10.000 & 9.700 & 11.155 & 5.000 & 4.000 & 9.000 & \\
\hline $500 \mathrm{mg} \times 3$ & & 15.000 & 14.550 & 16.733 & 7.500 & 6.000 & 13.500 & \\
\hline \multicolumn{8}{|c|}{ CEFETAMET PIVOXIL } & \\
\hline $500 \mathrm{mg} \times 2$ & & 7.735 & 7.503 & 8.628 & 3.866 & 4.000 & 7.866 & \\
\hline $500 \mathrm{mg} \times 3$ & & 11.600 & 11.252 & 12.940 & 5.800 & 6.000 & 11.800 & \\
\hline \multicolumn{8}{|c|}{ CLARITROMICINA } & \\
\hline $500 \mathrm{mg} \times 2$ & & 12.420 & 12.047 & 13.855 & 9.210 & 4.000 & 13.210 & \multirow{9}{*}{$\begin{array}{l}\text { Tabella } 6 \\
\text { Posologie giornalie- } \\
\text { re e relativi costi } \\
\text { (Lire) di trattamento } \\
\text { domiciliare ed } \\
\text { ospedaliero di alcu- } \\
\text { ni antibiotici orali } \\
\text { utilizzabili nella te- } \\
\text { rapia della CAP. }\end{array}$} \\
\hline \multicolumn{8}{|c|}{ CIPROFLOXACINA } & \\
\hline $250 \mathrm{mg} \times 2$ & & 5.800 & 5.626 & 6.470 & 2.900 & 4.000 & 6.900 & \\
\hline $500 \mathrm{mg} \times 2$ & & 11.335 & 10.995 & 12.644 & 5.670 & 4.000 & 9.670 & \\
\hline $750 \mathrm{mg} \times 2$ & & 16.850 & 16.345 & 18.796 & 8.425 & 4.000 & 12.425 & \\
\hline \multicolumn{8}{|c|}{ LEVOFLOXACINA } & \\
\hline \multicolumn{2}{|c|}{$250 \mathrm{mg} \times 2$ os } & 12.200 & 11.834 & 13.609 & 6.110 & 4.000 & 10.110 & \\
\hline \multicolumn{2}{|c|}{$500 \mathrm{mg} \times 1$ os } & 9.810 & 9.516 & 10.943 & 4.905 & 2.000 & 6.905 & \\
\hline \multicolumn{2}{|c|}{$500 \mathrm{mg} \times 2$ os } & 19.620 & 19.031 & 21.886 & 9.810 & 4.000 & 13.810 & \\
\hline
\end{tabular}

* Costo al pubblico scontato del 3\% $\quad$ Ipotizzato pari al 15\% $\$$ Abbiamo ipotizzato un costo di lavoro del personale pari a L. 2.000 per somministrazione

tipo di consumo. In altri casi sono state stimate di soggetti a basso rischio di morte (Classe Fine le unità consumate durante il percorso o fasi 1-3) fossero utilizzati singoli farmaci orali o del percorso terapeutico del paziente (es. nu- parenterali, mentre nei soggetti a rischio elevamero di visite mediche).

L'intero episodio di polmonite è stato suddiviso in una fase iniziale di terapia, in una fase finale ed in periodi aggiuntivi correlabili ad eventuali recidive o ricoveri per infezioni nosocomiali. Infine è stato conteggiato anche un periodo di convalescenza.

Nella tabella 5 sono riportati i valori base, nonchè i valori minimi e massimi utilizzati nell'analisi di sensibilità, relativi alle voci di costo previste dal modello a carico del SSN o della società ed a carico dell'ospedale.

In questo lavoro non abbiamo voluto confrontare due o più antibiotici particolari: nostro obiettivo principale era analizzare quale peso avessero i principali nodi decisionali che si incontrano nel percorso terapeutico di un paziente affetto da CAP. Pertanto i costi della terapia antibiotica, che abbiamo assunto nel modello, sono costi tendenziali "medi" ricavati da una valutazione non esaustiva, ma sufficientemente ampia, delle alternative disponibili sul mercato e più frequentemente utilizzate, anche in base alle indicazioni delle linee guida internazionali. Inoltre, abbiamo ipotizzato che nel caso to di morte fossero utilizzati associazioni di farmaci (es., una betalatamina + un macrolide). Infine per i pazienti ricoverati in UTI abbiamo ipotizzato l'uso di associazioni dei farmaci più potenti ed anche più costosi.

Nelle tabelle 6 e 7 sono riportati i costi giornalieri di trattamento domiciliare ed ospedaliero con alcuni antibiotici rispettivamente orali ed iniettivi che abbiamo considerato per stimare i valori assunti nel modello. Per ogni antibiotico sono considerate le posologie principali, il costo di acquisto per il SSN e per l'ospedale, l'eventuale costo aggiuntivo dei materiali necessari per la somministrazione parenterale, e, nel caso di trattamento in regime di ricovero, anche il costo del lavoro del personale ospedaliero. Nel caso di terapia antibiotica iniettiva a domicilio abbiamo considerato un costo di acquisto dell' antibiotico pari a quello ospedaliero, ipotizzando che tali trattamenti vengano effettuati da strutture ADI; il costo del personale è stato, invece, contabilizzato a parte come costo delle visite mediche ed infermieristiche.

Nel modello la dimensione temporale delle 


\section{Tabella 7}

Posologie giornaliere e relativi costi (Lire) di trattamento domiciliare ed ospedaliero di alcuni antibiotici iniettivi utilizzabili nella terapia della CAP.

\begin{tabular}{|c|c|c|c|c|c|c|}
\hline Antibiotico Parenterale & \multicolumn{2}{|c|}{ Acquisto antibiotico } & \multicolumn{2}{|c|}{$\begin{array}{l}\text { Soggetti in TAID } \\
\text { Costo per SSN }\end{array}$} & \multicolumn{2}{|c|}{$\begin{array}{l}\text { Soggetti Ricoverati } \\
\text { Costo per Ospedale }\end{array}$} \\
\hline $\begin{array}{ll}\text { Antibiotico } & \begin{array}{l}\text { Posologia } \\
\text { giornaliera }\end{array} \\
\end{array}$ & $\begin{array}{r}\text { Prezzo } \\
(\text { Lire }) \\
\end{array}$ & $\begin{array}{c}\text { Costo } \\
\text { ospedaliero }\end{array}$ & Materiali & $\begin{array}{c}\text { Totale } \\
\text { Domicilio } \\
\end{array}$ & $\begin{array}{l}\text { Mat+ } \\
\text { Lav* }\end{array}$ & $\begin{array}{c}\text { Totale } \\
\text { Ospedale } \\
\end{array}$ \\
\hline \multicolumn{7}{|c|}{ AMOXICILLINA/CLAV } \\
\hline $1,2 \mathrm{~g} \times 3$ iv & 36.000 & 18.000 & 9.000 & 27.000 & 36.000 & 54.000 \\
\hline $2,2 \mathrm{gx} 3$ iv & 52.500 & 26.250 & 9.000 & 35.250 & 36.000 & 62.250 \\
\hline \multicolumn{7}{|l|}{ PIP/TAZOBACTAM } \\
\hline $4,5 \mathrm{~g} \times 2 \mathrm{iv}$ & 112.400 & 56.200 & 6.000 & 62.200 & 24.000 & 80.200 \\
\hline $2,250 \mathrm{~g} \times 3 \mathrm{im}$ & 80.100 & 40.050 & 3.000 & 43.050 & 15.000 & 55.050 \\
\hline \multicolumn{7}{|l|}{ CEFTRIAXONE } \\
\hline $1 \mathrm{~g} \mathrm{x} 1$ iv & 36.200 & 18.100 & 3.000 & 21.100 & 12.000 & 30.100 \\
\hline $\lg \times 1 \mathrm{im}$ & 28.900 & 14.450 & 1.000 & 15.450 & 5.000 & 19.450 \\
\hline $2 \mathrm{~g} \mathrm{x} 1 \mathrm{iv}$ & 71.000 & 35.500 & 3.000 & 38.500 & 12.000 & 47.500 \\
\hline $1 \mathrm{~g} \mathrm{x} 2$ iv & 72.400 & 36.200 & 6.000 & 42.200 & 24.000 & 60.200 \\
\hline $\lg \times 2 \mathrm{im}$ & 57.800 & 28.900 & 2.000 & 30.900 & 10.000 & 38.900 \\
\hline \multicolumn{7}{|l|}{ CEFOTAXIME } \\
\hline $1 \mathrm{~g} \times 2 \mathrm{im}$ & 31.000 & 15.500 & 2.000 & 17.500 & 10.000 & 25.500 \\
\hline $1 \mathrm{~g} \times 3 \mathrm{im}$ & 46.500 & 23.250 & 3.000 & 26.250 & 15.000 & 38.250 \\
\hline $2 \mathrm{~g} \mathrm{x} 2$ iv & 62.000 & 31.000 & 6.000 & 37.000 & 24.000 & 55.000 \\
\hline $2 \mathrm{~g} \mathrm{x} 3 \mathrm{iv}$ & 93.000 & $4 \quad 46.500$ & 9.000 & 255.500 & 36.000 & 82.500 \\
\hline \multicolumn{7}{|l|}{ MEROPENEM } \\
\hline $500 \mathrm{mg} \times 3$ iv & 170.700 & 85.350 & 9.000 & 94.350 & 36.000 & 121.350 \\
\hline $1 \mathrm{~g} \times 2$ iv & 212.200 & 106.100 & 6.000 & 112.100 & 24.000 & 130.100 \\
\hline \multicolumn{7}{|l|}{ CLARITROMICINA } \\
\hline $0,5 \mathrm{~g} \times 1 \mathrm{iv}$ & 33.300 & 16.650 & 3.000 & 19.650 & 12.000 & 28.650 \\
\hline $\lg \times 1$ & 666.600 & 33.300 & 3.000 & 36.300 & 12.000 & 45.300 \\
\hline \multicolumn{7}{|l|}{ CIPROFLOXACINA } \\
\hline $200 \mathrm{mg} \times 1$ iv & 62.100 & 31.050 & 3.000 & 34.050 & 12.000 & 43.050 \\
\hline $200 \mathrm{mg} \times 2$ iv & 124.200 & 62.100 & 6.000 & 68.100 & 24.000 & 86.100 \\
\hline $400 \mathrm{mg} \mathrm{x} 1$ iv & \begin{tabular}{|l|}
130.100 \\
\end{tabular} & 65.050 & 3.000 & 68.050 & 12.000 & 77.050 \\
\hline $400 \mathrm{mg} \times 2$ iv & 260.200 & 131.100 & 6.000 & 137.100 & 24.000 & 155.100 \\
\hline $400 \mathrm{mg} \mathrm{x} 3$ iv & 390.300 & 195.150 & 9.000 & 204.150 & 36.000 & 231.150 \\
\hline \multicolumn{7}{|l|}{ LEVOFLOXACINA } \\
\hline $500 \mathrm{mg} \times 1$ iv & 119.000 & 59.500 & 3.000 & 62.500 & 12.000 & 71.500 \\
\hline $500 \mathrm{mg} \times 2$ iv & 238.000 & 119.000 & 6.000 & 125.000 & 24.000 & 143.000 \\
\hline \multicolumn{7}{|l|}{ AMIKACINA } \\
\hline $500 \mathrm{mg} \times 2$ iv & 25.800 & 12.900 & 6.000 & 18.900 & 24.000 & 36.900 \\
\hline $500 \mathrm{mg} \times 2 \mathrm{im}$ & 25.800 & 12.900 & 2.000 & 14.900 & 10.000 & 22.900 \\
\hline $\lg \times 1$ iv & 26.700 & 13.350 & 3.000 & 16.350 & 12.000 & 25.350 \\
\hline $\lg \times 1 \mathrm{im}$ & 26.700 & 13.350 & 1.000 & 14.350 & 5.000 & 18.350 \\
\hline \multicolumn{7}{|l|}{ TEICOPLANINA } \\
\hline $200 \mathrm{mg} \times 1$ iv & 83.000 & 41.550 & 3.000 & 44.550 & 12.000 & 53.550 \\
\hline $400 \mathrm{mg} x 1$ iv & 166.200 & 83.100 & 3.000 & 86.100 & 12.000 & 95.100 \\
\hline
\end{tabular}

*Come costo materiali abbiamo ipotizzato L. 1.000 per iniezione im e L. 3.000 per somministrazione iv. Come costo lavoro abbiamo ipotizzato L. 4.000 per iniezione im e L. 9.000 per somministrazione iv. 
succesive fasi della terapia e della malattia è stata considerata come variabile interindividuale e stimata mediante distribuzioni di frequenza, le principali delle quali sono riportate nelle tabelle 812. Nella valutazione di base sono stati utilizzati i valori mediani di ciascuna distribuzione, mentre nell' analisi mediante il metodo Monte Carlo ad ogni individuo sono stati attribuiti specifici intervalli di tempo campionati a caso dalle distribuzioni secondo le relative frequenze.

Infine al modello è stato fornito il valore ipotizzato per le variabili accessorie di costo specificate in tabella 13. Infatti, è verosimile che alcuni tipi di risorse, come l'utilizzo di ambulanze, interessi solo una frazione dei pazienti op- pure una frazione dei giorni di una determinata fase del trattamento.

\section{Valutazione di efficacia}

Abbiamo deciso di valutare l'efficacia utilizzando come parametro utile la percentuale di pazienti guariti, ovvero di sopravvissuti, al termine del ciclo temporale previsto dal modello. La quota complementare di pazienti è quella dei soggetti deceduti per effetto diretto o indiretto della polmonite. Il modello non prevedeva altre categorie di outcome clinici.

In realtà, le varie percentuali di guariti e di morti riflettono la combinazione degli effetti di molteplici cause tra cui le principali sono il tipo

\begin{tabular}{|cccc}
\hline Giorni per stabilizzare & FINE I $^{\circ}$-III & FINE IV $^{\circ}$ & FINE V $^{\circ}$ \\
\hline 1 & 0,02 & & \\
\hline 2 & 0,30 & 0,35 & 0,60 \\
\hline 3 & 0,35 & 0,45 & 0,40
\end{tabular}

\section{Tabella 8}

Distribuzione delle probabilità di raggiungere la stabilizzazione clinica con la terapia antibiotica iniziale in funzione dei giorni necessari.

\begin{tabular}{|cccc}
\hline $\begin{array}{c}\text { Giorni } \\
\text { Fase finale terapia }\end{array}$ & ${\text { FINE } \text { I }^{\circ} \text { III }^{\circ}}$ & FINE IV $^{\circ}$ & FINE V $^{\circ}$ \\
\hline 4 & 0,153 & 0,082 & 0,136 \\
\hline 5 & 0,170 & 0,179 & 0,255 \\
\hline 6 & 0,182 & 0,217 & 0,192 \\
\hline 7 & 0,166 & 0,175 & 0,156 \\
\hline 8 & 0,126 & 0,133 & 0,104 \\
\hline 9 & 0,084 & 0,088 & 0,068 \\
\hline 10 & 0,054 & 0,057 & 0,042 \\
\hline 11 & 0,034 & 0,035 & 0,026 \\
\hline 12 & 0,020 & 0,022 & 0,015 \\
\hline 13 & 0,011 & 0,012 & 0,006
\end{tabular}

\section{Tabella 9}

Distribuzioni delle probabilità di durata (giorni) della terapia antibiotica nella fase finale della trattamento nelle differenti classi di rischio di morte secondo Fine.

\begin{tabular}{|cccc}
\hline Giorni in UTI & FINE I $^{\circ}$ III $^{\circ}$ & FINE IV $^{\circ}$ & FINE V $^{\circ}$ \\
\hline 2 & 0,10 & & \\
\hline 3 & & 0,10 & \\
\hline 4 & 0,15 & & 0,10 \\
\hline 5 & 0,20 & & 0,15 \\
\hline 6 & 0,25 & 0,16 & 0,20 \\
\hline 7 & 0,2 & 0,20 & 0,25 \\
\hline 8 & & 0,25 & \\
\hline 9 & 0,10 & & 0,20 \\
\hline 10 & & 0,20 & 0,10
\end{tabular}

\section{Tabella 10}

Distribuzione delle probabilità di durata (giorni) della degenza in UTI nelle differenti classi di rischio di morte secondo Fine. 
Tabella 11

Distribuzione delle probabilità di alcuni parametri temporali complementari previsti dal modello decisionale.

\section{Tabella 12}

Distribuzione delle probabilità di prolungamento della degenza dopo aver ultimato la terapia antibiotica in gruppi di pazienti che presentano una diversa evoluzione clinica.

\section{Tabella 13}

Valori attribuiti ad alcune variabili accessorie di costo del modello.

\begin{tabular}{|ccccc}
\hline Giorni & $\begin{array}{c}\text { Distribuzione } \\
\text { probabilità giorni } \\
\text { terapia post- } \\
\text { stabilizzazione in } \\
\text { ricovero breve }\end{array}$ & $\begin{array}{c}\text { Distribuzione } \\
\text { probabilità } \\
\text { giorni di terapia } \\
\text { domiciliare post- } \\
\text { ricovero breve }\end{array}$ & $\begin{array}{c}\text { Distribuzione } \\
\text { probabilità } \\
\text { giorni di terapia } \\
\text { dopo cambio di } \\
\text { antibiotico }\end{array}$ & $\begin{array}{c}\text { Distribuzione } \\
\text { probabilità } \\
\text { durata ricovero } \\
\text { dopo iniziale } \\
\text { terapia } \\
\text { domiciliare }\end{array}$ \\
\hline 1 & 0,6 & & & \\
\hline 2 & 0,35 & 0,1 & 0,02 & \\
\hline 3 & 0,05 & 0,15 & 0,03 & 0,02 \\
\hline 4 & 0,25 & 0,1 & 0,03 \\
\hline 5 & 0,2 & 0,16 & 0,1 \\
\hline 6 & 0,15 & 0,2 & 0,16 \\
\hline 7 & 0,1 & 0,18 & 0,2 \\
\hline 8 & 0,05 & 0,12 & 0,12 \\
\hline 9 & & 0,08 & 0,08 \\
\hline 10 & & 0,05 & 0,05 \\
\hline 11 & & 0,03 & 0,03 \\
\hline 12 & & 0,02 & 0,02 \\
\hline 13 & & 0,006 & 0,006 \\
\hline 14 & & 0,004 & 0,004 \\
\hline 15 & & & \\
\hline 16 & & & \\
\hline 17 & & & \\
\hline
\end{tabular}

\begin{tabular}{|cccc}
\hline Giorni extra-terapia & $\begin{array}{c}\text { Soggetti che } \\
\text { stabilizzano }\end{array}$ & $\begin{array}{c}\text { Soggetti che non } \\
\text { stabilizzano e } \\
\text { cambiano antibiotico }\end{array}$ & Soggetti inviati in UTI \\
\hline 1 & 0,60 & 0,10 & \\
\hline 2 & 0,30 & 0,30 & 0,10 \\
\hline 3 & 0,10 & 0,40 & 0,20 \\
\hline 4 & & 0,15 & 0,40 \\
\hline 5 & & 0,05 & 0,15 \\
\hline 6 & & 0,10 \\
\hline 7 & & & 0,05
\end{tabular}

\begin{tabular}{|lccc}
\hline \multicolumn{1}{|c}{ Altre variabili di costo del modello } & $\begin{array}{c}\text { Valore } \\
\text { base }\end{array}$ & Min & Max \\
\hline $\begin{array}{l}\text { Frazione dei giorni di ricovero in cui è intervenuta l'assistenza } \\
\text { integrativa }\end{array}$ & 0,50 & 0,30 & 0,80 \\
\hline $\begin{array}{l}\text { Frazione di morti tra coloro che cambiano terapia, sul totale } \\
\text { della classe di rischio }\end{array}$ & 0,020 & 0,005 & 0,040 \\
\hline Frazione di morti precoci sul totale della classe di rischio & 0,05 & 0,01 & 0,20 \\
\hline $\begin{array}{l}\text { Frazioni di morti tardive in soggetti stabilizzati, sul totale della } \\
\text { classe di rischio }\end{array}$ & 0,01 & 0,001 & 0,05 \\
\hline Frazione di morti in UTI sul totale della classe di rischio & 0,90 & 0,70 & 0,95 \\
\hline $\begin{array}{l}\text { Frazione di ricoveri in cui è stato utilizzato il servizio } \\
\text { ambulanza }\end{array}$ & 0,20 & 0,05 & 0,40
\end{tabular}




\begin{tabular}{|c|c|c|c|c|c|c|}
\hline \multirow{3}{*}{ PERCORSI } & \multicolumn{5}{|c|}{$\begin{array}{l}\text { COSTO MEDIO/PAZIENTE TRATTATO } \\
\text { A CARICO DELLA SOCIETA'(Lire x 1.000) }\end{array}$} & \\
\hline & \multicolumn{5}{|c|}{ Rischio di morte (Classe a fine) } & \\
\hline & $\mathrm{I}^{\circ}$ & $\mathrm{II}^{\circ}$ & $\mathrm{III}^{\circ}$ & $\mathrm{IV}^{\circ}$ & $\mathrm{V}^{\circ}$ & \\
\hline \multicolumn{7}{|l|}{ RICOVERO IN OSPEDALE } \\
\hline \multicolumn{7}{|l|}{ ANTIBIOTICO PARENTERALE } \\
\hline \multicolumn{7}{|l|}{ Stabilizza - NO-Switch } \\
\hline Ricovero Breve & 6.045 & 6.045 & 6.045 & 6.210 & 6.210 & \\
\hline Ricovero Lungo & 5.887 & 6.018 & 6.150 & 6.563 & 6.705 & \\
\hline \multicolumn{7}{|l|}{ Stabilizza - Switch } \\
\hline Ricovero Breve & 5.817 & 5.817 & 5.817 & 5.884 & 5.884 & \\
\hline Ricovero Lungo & 5.887 & 6.018 & 6.160 & 6.563 & 6.705 & \\
\hline Cambia antibiotico & 6.279 & 6.416 & 6.556 & 6.718 & 6.919 & \\
\hline Invio in UTI & 9.193 & 9.297 & 9.402 & 9.899 & 10.031 & \\
\hline Morte precoce & 4.852 & 4.852 & 4.852 & 4.852 & 4.852 & \\
\hline \multicolumn{7}{|l|}{ ב ANTIBIOTICO ORALE } \\
\hline Stabilizza - Ricovero Breve & 5.817 & 5.817 & 5.817 & 5.885 & 5.885 & \\
\hline Stabilizza - Ricovero Lungo & 5.887 & 6.018 & 6.160 & 6.563 & 6.705 & \\
\hline Cambia antibiotico & 6.279 & 6.416 & 6.556 & 6.718 & 6.919 & \\
\hline Invio in UTI & 9.193 & 9.297 & 9.402 & 9.899 & 10.031 & \\
\hline Morte precoce & 4.852 & 4.852 & 4.852 & 4.852 & 4.852 & \\
\hline \multicolumn{7}{|l|}{ TERAPIA DOMICILIARE } \\
\hline \multicolumn{7}{|l|}{ ANTIBIOTICO PARENTERALE } \\
\hline Stabilizza - NO-Switch & 2.016 & 2.399 & 2.572 & 3.553 & 3.758 & \multirow{10}{*}{$\begin{array}{l}\text { Tabella } 14 \\
\text { Costo medio/pazien- } \\
\text { te trattato a carico } \\
\text { della società in fun- } \\
\text { zione del livello di } \\
\text { rischio di morte e } \\
\text { del percorso } \\
\text { terapeutico. }\end{array}$} \\
\hline Stabilizza - Switch & 1.743 & 2.081 & 2.208 & 2.689 & 2.816 & \\
\hline Cambia antibiotico & 2.747 & 3.180 & 3.402 & 3.946 & 4.391 & \\
\hline Ricovero dopo iniziale insuccesso & 8.453 & 8.663 & 8.664 & 6.584 & 6.584 & \\
\hline Morte precoce & 719 & 929 & 929 & 1.028 & 1.028 & \\
\hline ANTIBIOTICO ORALE & & & & & & \\
\hline Stabilizza & 1.607 & 1.944 & 2.072 & 2.643 & 2.783 & \\
\hline Cambia antibiotico & 2.155 & 2.523 & 2.681 & 3.036 & 3.351 & \\
\hline Ricovero dopo iniziale insuccesso & 8.317 & 8.527 & 8.528 & 6.389 & 6.389 & \\
\hline Morte precoce & 582 & 792 & 792 & 833 & 833 & \\
\hline
\end{tabular}

di infezione, le caratteristiche demografiche, socio-culturali e cliniche del paziente e l'efficacia del farmaco.

La mortalità non sembra essere influenzata dalla terapia farmacologia. D'altra parte, oggi, abbiamo a disposizione un notevole numero di antibiotici molto efficaci in grado di indurre il massimo risultato clinico ottenibile con la terapia antibiotica. Inoltre, tutti i trias clinici controllati sono finalizzati a dimostrare l'equivalenza terapeutica tra due antibiotici, ovvero a dimostrare che entrambi gli antibiotici sono in grado di guarire la massima percentuale possibile dei pazienti affetti da una data infezione. Poiché nel caso della polmonite non è ammissibile, per motivi etici, effettuare confronti versus placebo, ne consegue che non possiamo stabilire quale sia l'efficacia incrementale della tera- pia antibiotica nella CAP. La percentuale di guariti e di morti rappresenta un dato robusto e disponibile in letteratura e riteniamo sia utilizzabile come indice di efficacia in analisi di costo-efficacia o di minimizzazione dei costi.

\section{E. Indicatori utilizzati}

Abbiamo stimato il costo medio per paziente trattato e il rapporto costo/efficacia espresso come costo medio/paziente guarito. Tali parametri sono stati calcolati sull'intera popolazione simulata e su sottogruppi omogenei per tipo di percorso terapeutico.

\section{F. Software}

Il modello di albero decisionale e le analisi attuate sono state realizzate mediante il programma DATA 3.5 (TreeAge Co.). 
Tabella 15

Costo medio/paziente trattato a carico del SSN in funzione del livello di rischio di morte e del percorso terapeutico.

\begin{tabular}{|c|c|c|c|c|c|}
\hline \multirow{3}{*}{ PERCORSI } & \multicolumn{5}{|c|}{$\begin{array}{c}\text { COSTO MEDIO/PAZIENTE TRATTATO } \\
\text { A CARICO DEL SSN (Lire x } 1.000)\end{array}$} \\
\hline & \multicolumn{5}{|c|}{ Rischio di morte (Classe a fine) } \\
\hline & $\mathrm{I}^{\circ}$ & $\mathrm{II}^{\circ}$ & $\mathrm{III}^{\circ}$ & $\mathrm{IV}^{\circ}$ & $\mathrm{V}^{\circ}$ \\
\hline \multicolumn{6}{|l|}{ RICOVERO IN OSPEDALE } \\
\hline \multicolumn{6}{|l|}{ ANTIBIOTICO PARENTERALE } \\
\hline \multicolumn{6}{|l|}{ Stabilizza - NO-Switch } \\
\hline Ricovero Lungo & 4.568 & 4.568 & 4.579 & 4.590 & 4.600 \\
\hline \multicolumn{6}{|l|}{ Stabilizza - Switch - } \\
\hline Ricovero Lungo & 4.568 & 4.568 & 4.579 & 4.590 & 4.600 \\
\hline Cambia antibiotico & 4.576 & 4.586 & 4.588 & 4.568 & 4.551 \\
\hline Invio in UTI & 6.617 & 6.617 & 6.617 & 6.617 & 6.617 \\
\hline Morte precoce & 4.449 & 4.449 & 4.449 & 4.449 & 4.449 \\
\hline \multicolumn{6}{|l|}{ ANTIBIOTICO ORALE } \\
\hline Stabilizza - Ricovero Breve & 4.701 & 4.701 & 4.701 & 4.769 & 4.769 \\
\hline Stabilizza - Ricovero Lungo & 4.568 & 4.568 & 4.579 & 4.590 & 4.601 \\
\hline Cambia antibiotico & 4.576 & 4.586 & 4.588 & 4.568 & 4.551 \\
\hline Invio in UTI & 6.617 & 6.617 & 6.617 & 6.617 & 6.617 \\
\hline Morte precoce & 4.449 & 4.449 & 4.449 & 4.449 & 4.449 \\
\hline \multicolumn{6}{|l|}{ TERAPIA DOMICILIARE } \\
\hline \multicolumn{6}{|l|}{ ב ANTIBIOTICO PARENTERALE } \\
\hline Stabilizza - NO-Switch & 977 & 1.247 & 1.307 & 1.948 & 2.041 \\
\hline Stabilizza - Switch & 704 & 928 & 943 & 1.085 & 1.099 \\
\hline Cambia antibiotico & 1.594 & 1.914 & 2.024 & 2.342 & 2.560 \\
\hline Ricovero dopo iniziale insuccesso & 6.927 & 7.136 & 7.137 & 5.057 & 5.067 \\
\hline Morte precoce & 380 & 590 & 590 & 689 & 689 \\
\hline \multicolumn{6}{|l|}{ ANTIBIOTICO ORALE } \\
\hline Stabilizza & 567 & 792 & 806 & 1.038 & 1.066 \\
\hline Cambia antibiotico & 1.003 & 1.257 & 1.302 & 1.432 & 1.520 \\
\hline Ricovero dopo iniziale insuccesso & 6.790 & 6.999 & 7.001 & 4.862 & 4.862 \\
\hline Morte precoce & 244 & 454 & 454 & 494 & 494 \\
\hline
\end{tabular}

\section{RISULTATI}

I risultati dell' analisi di base sono riassunti nelle tabelle 14-24 e nella figura 4.

Le tabelle 14-15 riportano i valori che può assumere il costo medio per trattare un paziente, rispettivamente secondo la prospettiva della società e del SSN, in funzione dei differenti percorsi terapeutici attuabili e del livello di rischio di morte del paziente.

L'analisi conferma che nel complesso il ricovero ospedaliero comporta un costo sociale e sanitario nettamente superiore a quello correlato con il trattamento domiciliare. Nettamente inferiore, sebbene significativa, è la differenza dei costi, sia sociali sia sanitari, tra terapia parenterale e terapia orale, oppure tra pa- zienti per i quali si attua la switch therapy e quelli che continuano l'antibiotico parenterale, ed anche tra ricovero breve e ricovero lungo. I soggetti che non rispondono al trattamento antibiotico iniziale costano decisamente di più alla società ed al SSN.

Il livello del rischio di morte (classi di Fine), poiché è direttamente correlato con la gravità del paziente, condiziona la quantità di risorse consumate. Ne risentono soprattutto quei fattori di costo che intervengono nell' assistenza dei pazienti più impegnativi, come il ricovero in UTI, e l'utilizzo di antibiotici parenterali più potenti e costosi.

La tabella 16 riporta i valori che può assumere il costo medio di ricovero a carico dell'ospedale nell'ipotesi che siano validi i costi 
medi giornalieri di degenza imposti al modello. Com'è facilmente intuibile, ridurre i giorni di degenza comporta un notevole vantaggio per l'ospedale e può consentire di dimezzare quasi il costo/ricovero dei soggetti che hanno un buon andamento clinico. I soggetti, invece, che non rispondono positivamente alla terapia iniziale e soprattutto quelli che peggiorano e vengono inviati in UTI, rappresentano un aggravio particolarmente oneroso per l'ospedale.

La tabella 17 riporta i valori che può assumere il costo medio del trattamento antibiotico in ospedale. Il costo ospedaliero del trattamento antibiotico risente soprattutto della durata e del costo giornaliero di acquisto dei farmaci. Gli antibiotici orali, che in genere costano meno e non richiedono elevati costi aggiuntivi per la somministrazione, risultano particolarmente convenienti, rispetto a quelli iniettivi, soprattutto nei soggetti che hanno un andamento clinico favorevole.

Nel complesso il costo del trattamento antibiotico ospedaliero rappresenta una quota inferiore al $10 \%$ del costo globale di ricovero.

Viste a posteriori, le decisioni prese in una popolazione di pazienti appaiono come probabilità. Conoscendo la probabilità (frequenza) con cui viene presa una decisione, o un insieme di decisioni, in un dato sistema socio-assistenziale, si può stimare quali siano i costi totali ed i costi medi/paziente per aggregazioni di percorsi.

Le tabelle 18,19 e 20 riportano le valutazioni del costo medio per paziente trattato, attuate rispettivamente nella prospettiva della società, del SSN e dell'ospedale, ottenute con la simulazione del modello dopo aver sostituito i nodi decisionali con nodi probabilistici ed aver fornito le probabilità che avvenga una data scelta.

I nuovi valori del costo medio/paziente trattato così ottenuti risentono delle frequenze di ricovero, differente per ogni classe di rischio, nonché delle frequenze di scelta della via parenterale, della switch therapy e della dimissione precoce.

Il costo medio di trattamento a carico della società, del SSN o dell'ospedale, aumenta progressivamente con l'aumentare del rischio di morte, sia quando si considera l'intera frazione di pazienti aggregati in una medesima classe di Fine, sia quando si considerano sottogruppi aggregati, oltre che per livello di rischio, anche per tipo di percorso.

Mediante il metodo Monte Carlo abbiamo simulato i percorsi di 2000 ipotetici pazienti per verificare quale fosse il costo medio generale e la sua distribuzione nella prospettiva della società, del SSN e dell'ospedale. Il campione è stato ricostruito tenendo conto di tutte le distribuzioni e di tutte le probabilità che costituiscono la struttura del modello decisionale.

I risultati sono riportati nella figura 4 e nella tabella 21. Nessuna delle tre distribuzioni si presenta regolare e simmetrica. La distribuzione del costo/paziente per la società appare bimodale con una estensione codata verso i valori elevati. La distribuzione del costo/paziente per il SSN si presenta trimodale: la compo-

\begin{tabular}{|c|c|c|c|c|c|}
\hline \multirow{3}{*}{ PERCORSI } & \multicolumn{5}{|c|}{$\begin{array}{c}\text { COSTO MEDIO TRATTAMENTO } \\
\text { ANTIBIOTICO IN OSPEDALE (Lire x 1.000) }\end{array}$} \\
\hline & \multicolumn{5}{|c|}{ Rischi di morte (Classe a fine) } \\
\hline & $\mathrm{I}^{\circ}$ & $\mathrm{II}^{\circ}$ & $\mathrm{III}^{\circ}$ & $\mathrm{IV}^{\circ}$ & $\mathrm{V}^{\circ}$ \\
\hline \multicolumn{6}{|l|}{ RICOVERO IN OSPEDALE } \\
\hline \multicolumn{6}{|l|}{ ANTIBIOTICO PARENTERALE } \\
\hline $\begin{array}{l}\text { Stabilizza - NO-Switch } \\
\text { Ricovero Breve }\end{array}$ & 206,0 & 206,0 & 206,0 & 372,0 & 372,0 \\
\hline Ricovero Lungo & 463,5 & 515,0 & 566,5 & $1.302,0$ & $1.395,0$ \\
\hline $\begin{array}{l}\text { Stabilizza - Switch - } \\
\text { Ricovero Breve }\end{array}$ & 164,5 & 164,5 & 164,5 & 289,0 & 289,0 \\
\hline Ricovero Lungo & 214,5 & 224,5 & 234,5 & 389,0 & 399,0 \\
\hline Cambia antibiotico & 805,5 & 898,5 & 991,5 & $1.302,0$ & $1.488,0$ \\
\hline Invio in UTI & $1.114,5$ & $1.114,5$ & $1.114,5$ & $1.559,0$ & $1.719,0$ \\
\hline Morte precoce & 154,5 & 154,5 & 154,5 & 279,0 & 279,0 \\
\hline \multicolumn{6}{|l|}{ ANTIBIOTICO ORALE } \\
\hline Stabilizza - Ricovero Breve & 40,0 & 40,0 & 40,0 & 86,0 & 86,0 \\
\hline Stabilizza - Ricovero Lungo & 90,0 & 100,0 & 110,0 & 301,0 & 322,5 \\
\hline Cambia antibiotico & 180,5 & 202,0 & 223,5 & 301,0 & 344,0 \\
\hline Invio in UTI & 990,0 & 990,0 & 990,0 & $1.344,5$ & $1.504,5$ \\
\hline Morte precoce & 30,0 & 30,0 & 30,0 & 64,5 & 64,5 \\
\hline
\end{tabular}

\section{Tabella 16}

Costo medio/paziente trattato a carico dell'ospedale in funzione del percorso e del livello di rischio di morte. 
nente a sinistra raggruppa tendenzialmente i corrisponde un minor numero di guariti e quinsoggetti trattati a domicilio, quella centrale i di il costo/paziente guarito aumenta sia nei soggetti trattati in ospedale o in ospedale e a sottogruppi trattati in ospedale sia in quelli tratdomicilio, la componente a destra i soggetti ri- tati a domicilio. Come sopra osservato, anche il coverati gravi che in genere finiscono in UTI. costo di trattamento aumenta in funzione del La distribuzione del costo/ricovero è sostan- livello di rischio di morte. Pertanto il rapporto zialmente bimodale ma fortemente asimmetrica. costo/efficacia aumenta con l'aumentare del ri-

Confrontando le statistiche delle tre distri- schio di morte con forti incrementi dovuti conbuzioni dei costi generati dal campione simula- temporaneamente all'aumento del numeratore to (tabella 21) si può osservare come il costo/ e alla diminuzione del denominatore. paziente per la società sia mediamente superiore di oltre 1,5 milioni di Lire rispetto al costo per il SSN.

Il costo medio di produzione del servizio ospedaliero, secondo la simulazione, sarebbe di circa 5,5 milioni di Lire, una cifra di poco superiore al valore medio delle tariffe DRG relative alla polmonite.

Le tabelle 22-24 riportano i valori che può assumere il costo medio/paziente guarito, rispettivamente secondo la prospettiva della società, del SSN e dell'ospedale, in funzione dei differenti percorsi terapeutici attuabili e del livello di rischio di morte del paziente. La stima è stata attuata sostituendo, come sopra, i nodi decisionali con nodi probabilistici al fine di rappresentare un profilo di ciò che si verifica in un dato contesto e fissare una realizzazione del sistema assistenziale da prendere come riferimento per eventuali interventi correttivi.

L'indice costo/efficacia risente ovviamente della frequenza dei successi: a rischi elevati

\section{ANALISI DI SENSIBILITÀ}

Per valutare la robustezza delle valutazioni di base attuate con il modello e stimare il peso di variabili alle quali è stato attribuito un valore incerto, abbiamo realizzato una serie di analisi di sensibilità.

La prima verifica è consistita nel valutare quale sarebbe la conseguenza di applicare rigorosamente l'indicazione di ricoverare solo i pazienti a rischio di morte medio o elevato (classe di Fine 4 e 5) e di trattare a domicilio tutti i pazienti di classe 1-3.

I risultati, espressi come costo medio per paziente trattato, sono riportati nella tabella 25.

L'applicazione integrale delle indicazioni che derivano dalla classificazione del rischio di morte porterebbe ad un risparmio medio di circa 950.000 Lire per paziente trattato a vantaggio della società, di circa 850.000 a vantaggio

Tabella 17

Costo medio del trattamento antibiotico dei pazienti ricoverati in ospedale in funzione del percorso e del livello di rischio di morte.

\begin{tabular}{|c|c|c|c|c|c|}
\hline \multirow[t]{2}{*}{ PERCORSI } & \multicolumn{5}{|c|}{$\begin{array}{c}\text { COSTO MEDIO TRATTAMENTO } \\
\text { ANTIBIOTICO IN OSPEDALE (Lire x 1.000) }\end{array}$} \\
\hline & FINE I $^{\circ}$ & $\begin{array}{c}\text { FINE } \\
\text { II }^{\circ}\end{array}$ & $\begin{array}{l}\text { FINE } \\
\text { III }^{\circ}\end{array}$ & $\begin{array}{l}\text { FINE } \\
\text { IV }^{\circ}\end{array}$ & $\begin{array}{l}\text { FINE } \\
\mathrm{V}^{\circ}\end{array}$ \\
\hline \multicolumn{6}{|l|}{ RICOVERO IN OSPEDALE } \\
\hline \multicolumn{6}{|l|}{ ANTIBIOTICO PARENTERALE } \\
\hline $\begin{array}{l}\text { Stabilizza - NO-Switch - } \\
\text { Ricovero Breve }\end{array}$ & 206,0 & 206,0 & 206,0 & 372,0 & 372,0 \\
\hline $\begin{array}{l}\text { Stabilizza - NO-Switch - } \\
\text { Ricovero Lungo }\end{array}$ & 463,5 & 515,0 & 566,5 & $1.302,0$ & $1.395,0$ \\
\hline $\begin{array}{l}\text { Stabilizza - Switch - } \\
\text { Ricovero Breve }\end{array}$ & 164,5 & 164,5 & 164,5 & 289,0 & 289,0 \\
\hline $\begin{array}{l}\text { Stabilizza - Switch - } \\
\text { Ricovero Lungo }\end{array}$ & 214,5 & 224,5 & 234,5 & 389,0 & 399,0 \\
\hline Cambia antibiotico & 805,5 & 898,5 & 991,5 & $1.302,0$ & $1.488,0$ \\
\hline Invio in UTI & $1.114,5$ & $1.114,5$ & $1.114,5$ & $1.559,0$ & $1.719,0$ \\
\hline Morte precoce & 154,5 & 154,5 & 154,5 & 279,0 & 279,0 \\
\hline \multicolumn{6}{|l|}{ ANTIBIOTICO ORALE } \\
\hline Stabilizza - Ricovero Breve & 40,0 & 40,0 & 40,0 & 86,0 & 86,0 \\
\hline Stabilizza - Ricovero Lungo & 90,0 & 100,0 & 110,0 & 301,0 & 322,5 \\
\hline Cambia antibiotico & 180,5 & 202,0 & 223,5 & 301,0 & 344,0 \\
\hline Invio in UTI & 990,0 & 990,0 & 990,0 & $1.344,5$ & $1.504,5$ \\
\hline Morte precoce & 30,0 & 30,0 & 30,0 & 64,5 & 64,5 \\
\hline
\end{tabular}


del SSN e di circa 1,4 milioni a vantaggio dell'ospedale. Tale risultato risulta dal fatto di spostare un notevole numero di soggetti dall' assistenza ospedaliera all' assitenza domiciliare e di spostare, invece, un minor numero di soggetti dal trattamento a domicilio al ricovero in ospedale. La tabella 25 evidenzia come, con la nuova strategia, diminuiscano i costi relativi ai pazienti di classe 1-3, mentre aumentano i costi relativi ai pazienti di classe 4 e 5 .

Alcuni dei risultati più significativi dell'analisi di sensibilità ad una via sono riportati nelle figure 5-6, ognuna delle quali è costituita da un diagramma "tornado" che rappresenta, in ordi- ne decrescente d'importanza, il peso che può avere la variazione di valore dei singoli parametri considerati sulla formazione del costo medio per paziente trattato. I parametri più sensibili sono quelli che inducono una più ampia escursione del costo medio.

Il costo medio di trattamento a carico della società (figura 5) risente principalmente della variazione del valore delle tariffe DRG dei ricoveri per polmonite e del valore della perdita di produttività. In quarta posizione, con un discreto peso, si colloca il costo dell' assistenza integrativa prestata dai familiari o dal volontariato. Un peso via via inferiore hanno il costo della

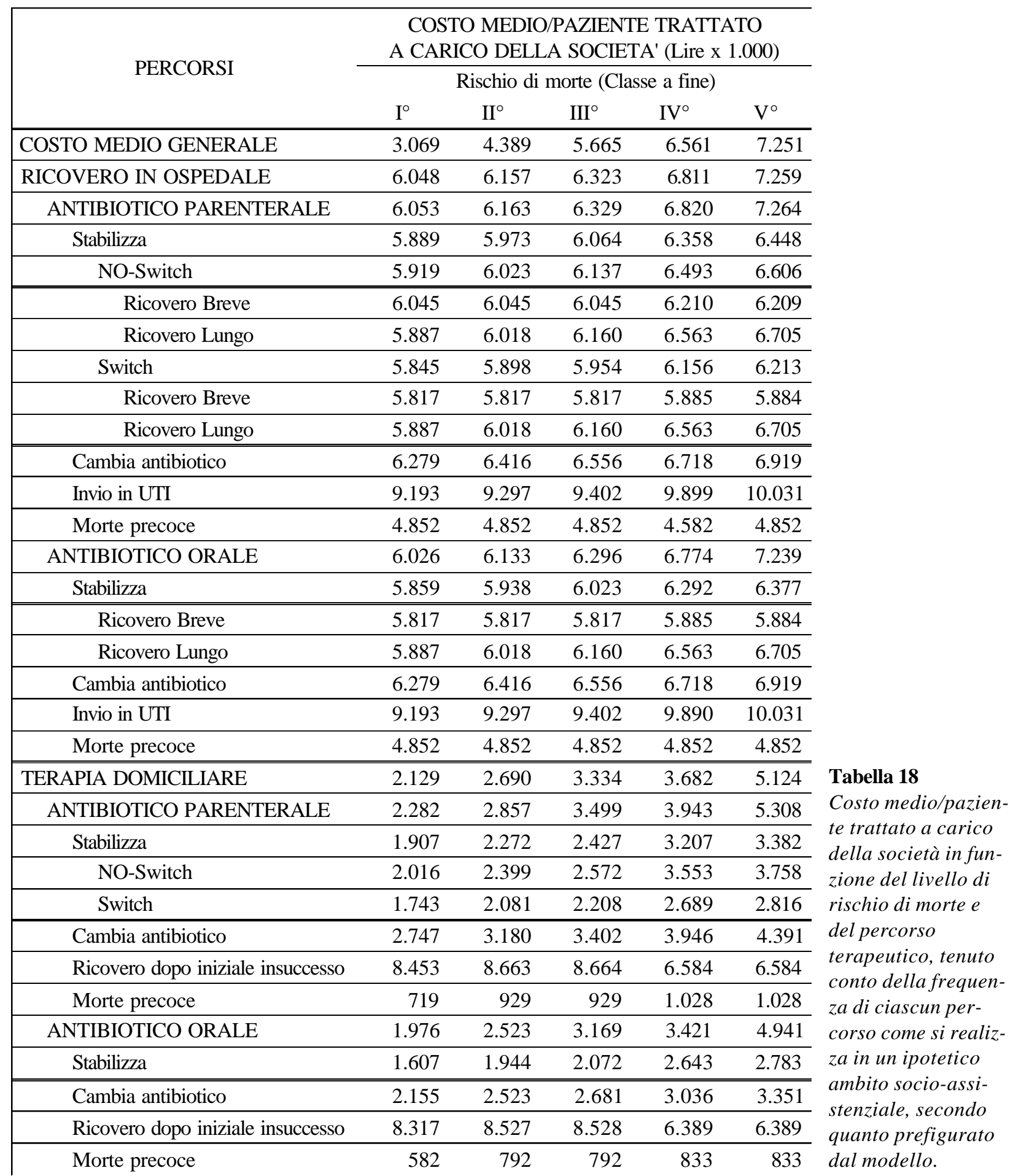


visita medica, il costo della terapia antibiotica parenterale a domicilio e l'entità delle piccole spese che i pazienti e i familiari sostengono per attuare la terapia della CAP. Le altre voci di costo considerate inducono una scarsa influenza sulla formazione del costo medio del trattamento della CAP a carico della società.

Il costo medio di trattamento a carico del SSN (figura 6) risente soprattutto del valore della tariffa DRG relativa ai ricoveri per polmonite non complicata. In seconda posizione, con un notevole peso, si colloca il valore del DRG per polmoniti complicate. Influenza inferiore hanno via via il costo della visita medica a do- micilio e della terapia antibiotica parenterale a domicilio. Le altre voci di costo hanno una minore importanza nella formazione del costo medio di trattamento a carico del SSN.

\section{DISCUSSIONE}

Abbiamo sviluppato un modello di albero decisionale che rappresenta i percorsi terapeutici attuabili dai pazienti affetti da CAP, dal momento in cui si pone diagnosi di sospetta CAP fino alla risoluzione del caso con la guarigione o con la morte. In particolare l'albero del-

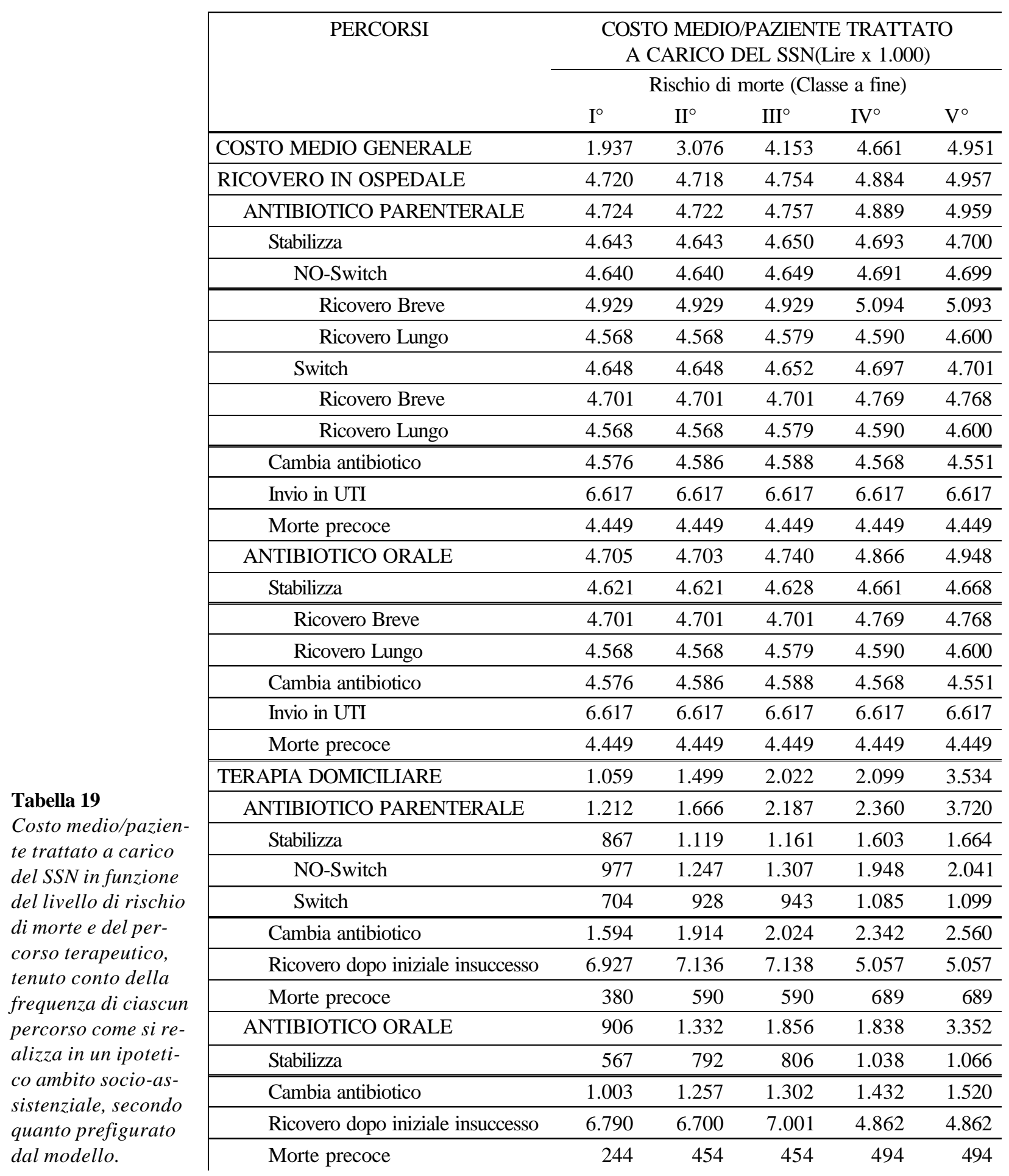




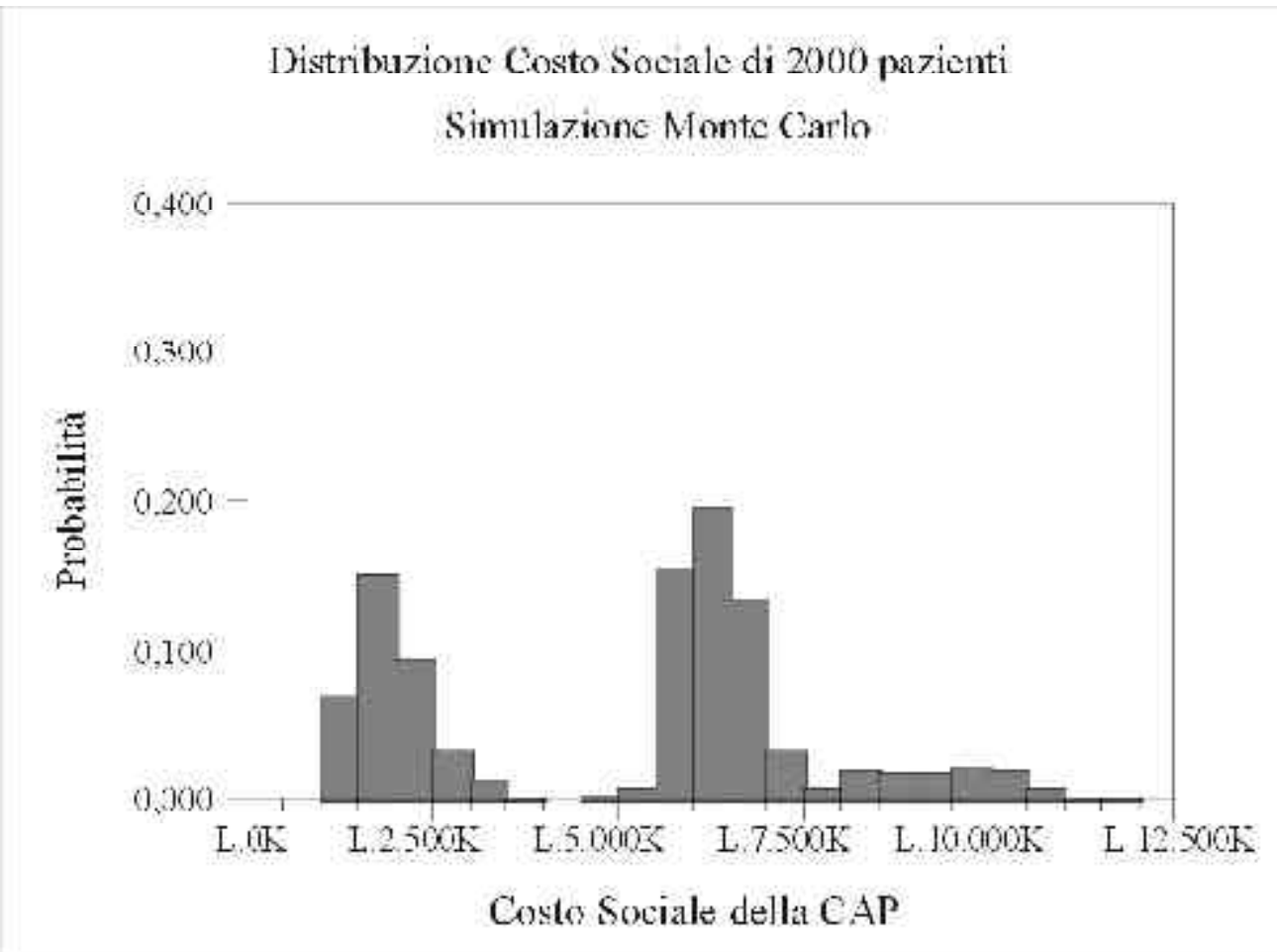

le decisioni considera il rischio di morte una delle variabili determinanti nell'indirizzare alcune fondamentali scelte strategiche, come il ricovero in ospedale e l'effettuazione di terapie antibiotiche più aggressive e prolungate.

Abbiamo alimentato il modello con dati ricavati dalla letteratura e con dati considerati ragionevoli in base ad un insieme di valutazioni e di analisi preliminari. La simulazione attuata ha avuto come scopo principale quello di dimostrare la validità e soprattutto l'utilità del modello implementato. I risultati ottenuti sono

probabilmente non lontani da quelli verificabili in un concreto sistema socio-sanitario, ma la controprova potrebbe derivare solo dall'utilizzo di dati direttamente ricavati dal sistema stesso che interessa indagare. I dati di mortalità e le frequenze di ricovero e di invio in UTI sono quelli della letteratura e riflettono la realtà degli Stati Uniti. Le altre variabili, che abbiamo dovuto ipotizzare per poter simulare il modello, sono tarate sulla situazione socio-assistenziale italiana. Analogamente, i valori monetari dei costi rispecchiano la realtà italiana e si riferi-

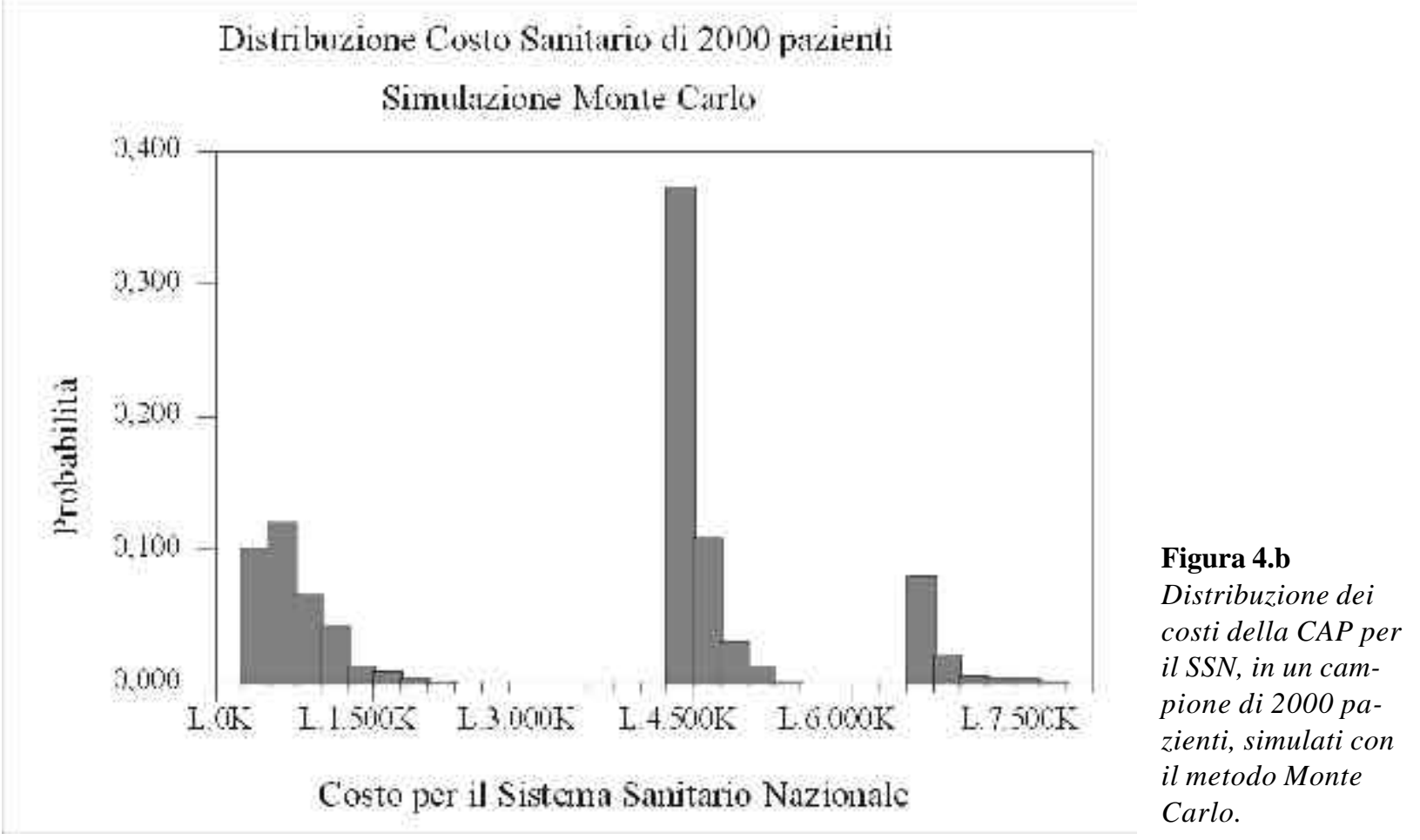

Figura 4.a

Distribuzione dei costi della CAP per la Società, in un campione di 2000 pazienti, simulati con il metodo Monte Carlo.
Distribuzione dei costi della CAP per li metodo Monte Carlo. 
Figura 4.c

Distribuzione dei costi della CAP per l'ospedale, in un campione di 2000 pazienti, simulati con il metodo Monte Carlo.
Tabella 20

Costo medio/paziente trattato a carico dell'ospedale in funzione del livello di rischio di morte e del percorso terapeutico, tenuto conto della frequenza di ciascun percorso come si realizza in un ipotetico ambito socio-assistenziale, secondo quanto prefigurato dal modello.
Distribuzione Costo por l'Crapedale di 2004 pazienti

Simulazione Monte Carlo

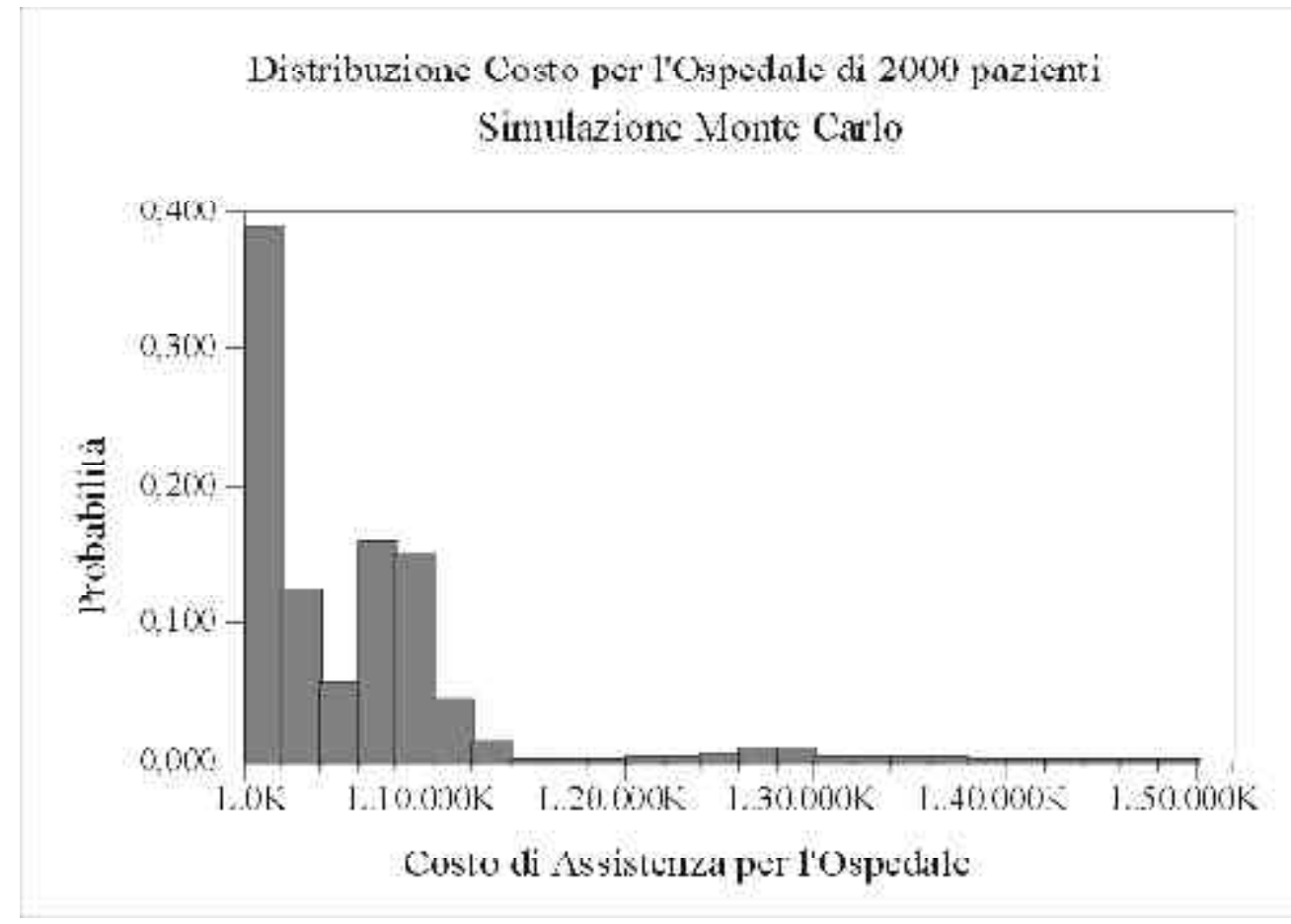

\begin{tabular}{|c|c|c|c|c|c|}
\hline \multirow{3}{*}{ PERCORSI } & \multicolumn{5}{|c|}{$\begin{array}{c}\text { COSTO MEDIO/PAZIENTE TRATTATO } \\
\text { A CARICO DELL'OSPEDALE (Lire x 1.000) }\end{array}$} \\
\hline & \multicolumn{5}{|c|}{ Rischio di morte (Classe a fine) } \\
\hline & $\mathrm{I}^{\circ}$ & $\mathrm{II}^{\circ}$ & III $^{\circ}$ & $\mathrm{IV}^{\circ}$ & $\mathrm{V}^{\circ}$ \\
\hline COSTO MEDIO GENERALE & 1.572 & 3.125 & 5.341 & 8.587 & 12.329 \\
\hline RICOVERO IN OSPEDALE & 5.257 & 5.767 & 6.545 & 9.225 & 12.365 \\
\hline ANTIBIOTICO PARENTERALE & 5.280 & 5.793 & 6.573 & 9.258 & 12.383 \\
\hline Stabilizza & 4.344 & 4.696 & 5.048 & 6.103 & 6.456 \\
\hline NO-Switch & 4.858 & 5.298 & 5.738 & 7.058 & 7.498 \\
\hline Ricovero Breve & 2.288 & 2.288 & 2.288 & 2.288 & 2.288 \\
\hline Ricovero Lungo & 5.500 & 6.050 & 6.600 & 8.250 & 8.800 \\
\hline Switch & 3.573 & 3.793 & 4.013 & 4.672 & 4.893 \\
\hline Ricovero Breve & 2.288 & 2.288 & 2.288 & 2.288 & 2.288 \\
\hline Ricovero Lungo & 5.500 & 6.050 & 6.600 & 8.250 & 8.800 \\
\hline Cambia antibiotico & 7.113 & 7.649 & 8.228 & 8.993 & 9.906 \\
\hline Invio in UTI & 22.480 & 22.920 & 23.360 & 29.350 & 31.850 \\
\hline Morte precoce & 1.650 & 1.650 & 1.650 & 1.650 & 1.650 \\
\hline ANTIBIOTICO ORALE & 5.164 & 5.665 & 6.435 & 9.091 & 12.292 \\
\hline Stabilizza & 4.215 & 4.545 & 4.875 & 5.865 & 6.195 \\
\hline Ricovero Breve & 2.288 & 2.288 & 2.288 & 2.288 & 2.288 \\
\hline Ricovero Lungo & 5.500 & 6.050 & 6.600 & 8.250 & 8.800 \\
\hline Cambia antibiotico & 7.113 & 7.649 & 8.228 & 8.993 & 9.906 \\
\hline Invio in UTI & 22.480 & 22.920 & 23.360 & 29.350 & 31.850 \\
\hline Morte precoce & 1.650 & 1.650 & 1.650 & 1.650 & 1.650 \\
\hline
\end{tabular}




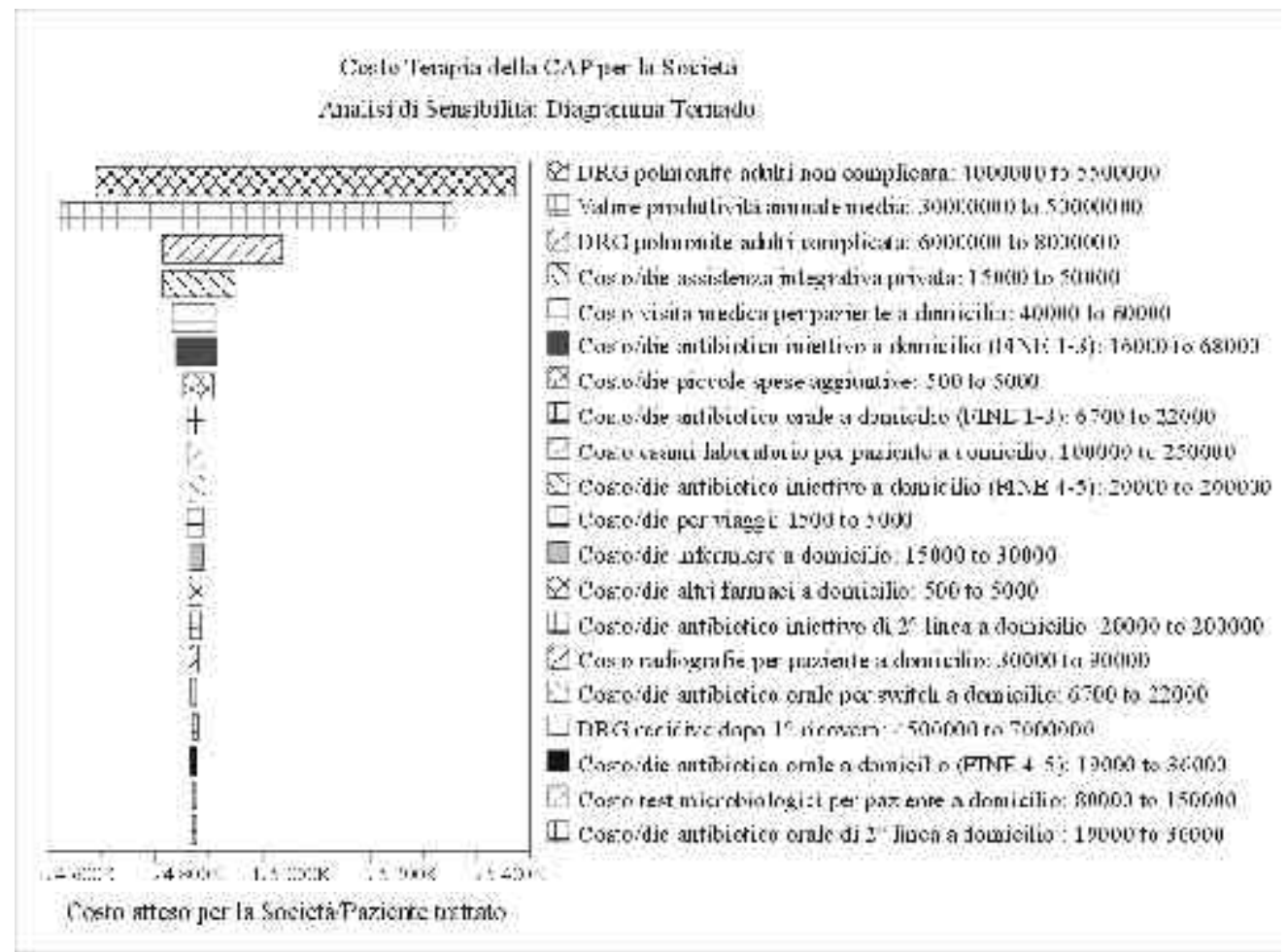

scono all'anno 2000.

Con il modello decisionale che abbiamo sviluppato è stato possibile stimare il costo medio per ciclo di trattamento della CAP e l'indice costo/efficacia espresso come costo medio per paziente guarito. Questi indicatori sono stati stimati in relazione ai diversi percorsi strategici e alle classi di rischio secondo Fine, considerando sia l'intera popolazione di pazienti affetti da CAP sia sottogruppi di dimensioni diverse. Questo tipo di analisi consente di verificare non solo quali siano i percorsi più problematici o più costosi, ma anche di rilevare quali parametri differenziali dei pazienti influenzino le scelte e siano rilevanti ai fini sanitari ed economici.
La struttura dell'albero ha consentito di verificare che $\mathrm{i}$ fattori di costo più importanti per il SSN sono i costi di ricovero, mentre i costi dell' assistenza domiciliare sono significativamente inferiori. Il costo della terapia antibiotica iniettiva, sia a domicilio che in ospedale, è significativamente più alta di quella orale. Tuttavia la differenza di costi tra antibiotico parenterale ed antibiotico orale è nettamente inferiore alla differenza di costi tra ricovero e terapia a domicilio.

Per l'ospedale è particolarmente conveniente ridurre le giornate di degenza, ognuna delle quali si stima possa costare dalle 400.000 alle 800.000 Lire per reparti di medicina e circa 2,5

\section{Figura 5}

Analisi di sensibilità ad una via dei principali parametri di costo che incidono sulla società. Diagramma "Tornado" calcolato sulla radice dell'albero delle decisioni, dove viene stimato il costo medio/paziente trattato a carico della società.

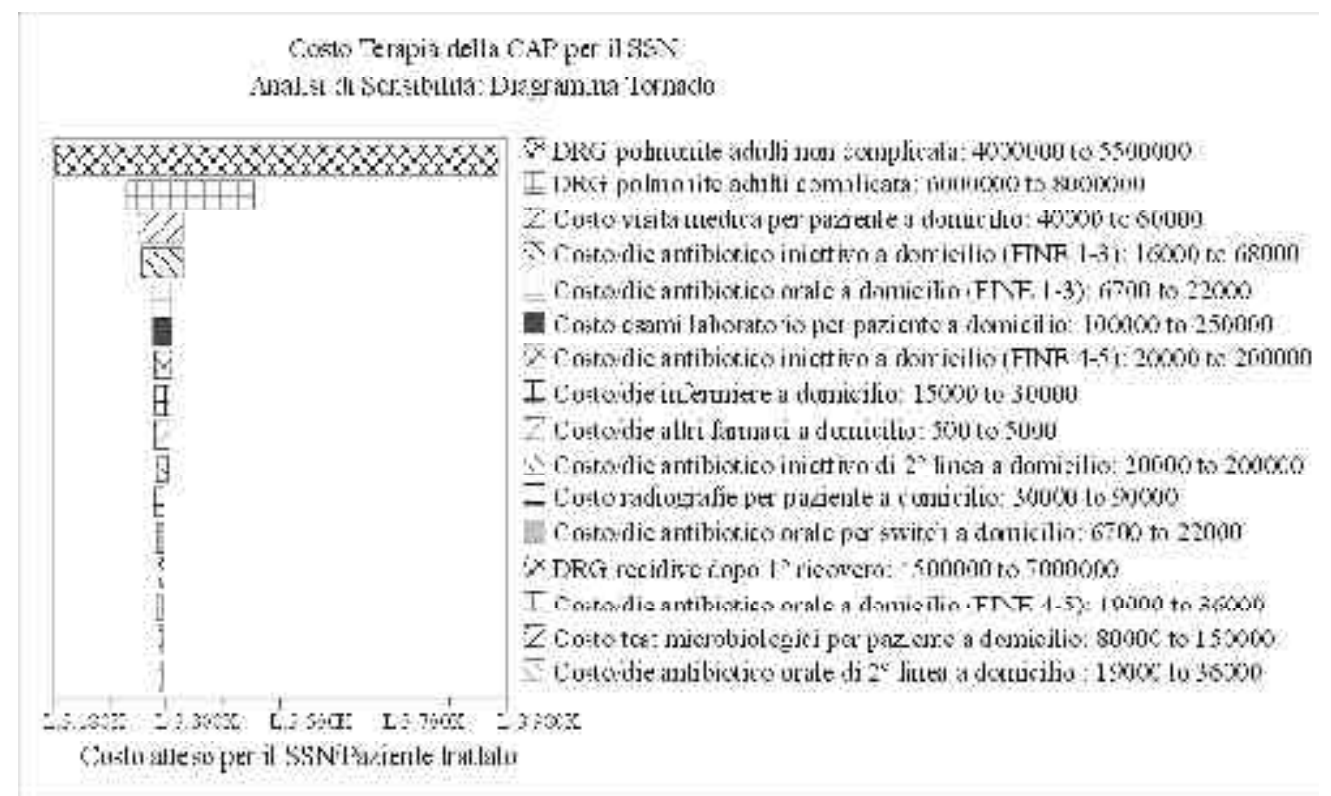

\section{Figura 6}

Analisi di sensibilità ad una via dei principali parametri di costo che incidono sul Sistema Sanitario Nazionale. Diagramma "Tornado" calcolato sulla radice dell' albero delle decisioni, dove viene stimato il costo medio/paziente trattato a carico del SSN. 
Tabella 21

Statistica della di-

stribuzione dei costi della CAP per il SSN, la Società e l'Ospedale in un campione di 2000 pazienti simulati con il metodo Monte Carlo.

\begin{tabular}{|lccc}
\hline & \multicolumn{2}{c}{ COSTO/PAZIENTE TRATTATO (Lire x 1.000) } \\
\cline { 2 - 4 } & SOCIETA' & SSN & OSPEDALE \\
\hline Media & 5.064 & 3.438 & 5.457 \\
\hline Moda & 6.161 & 4.449 & 7.164 \\
\hline SD & 2.592 & 2.140 & \\
\hline Minimo & 1.064 & 387 & 3.300 \\
\hline $5 \%$ & 1.447 & 445 & 20.750 \\
\hline Mediana $(50 \%)$ & 5.918 & 4.449 & 49.600
\end{tabular}

\begin{tabular}{|c|c|c|c|c|c|}
\hline \multirow{3}{*}{ PERCORSI } & \multirow{2}{*}{\multicolumn{5}{|c|}{$\begin{array}{l}\text { COSTO MEDIO/PAZIENTE GUARITO } \\
\text { A CARICO DELLA SOCIETA' (Lire x 1.000) } \\
\text { Rischio di morte (Classe a fine) }\end{array}$}} \\
\hline & & & & & \\
\hline & $\mathrm{I}^{\circ}$ & $\mathrm{II}^{\circ}$ & $\mathrm{III}^{\circ}$ & $\mathrm{IV}^{\circ}$ & $\mathrm{V}^{\circ}$ \\
\hline COSTO MEDIO GENERALE & 3.092 & 4.442 & 5.758 & 7.245 & 10.007 \\
\hline RICOVERO IN OSPEDALE & 6.104 & 6.240 & 6.430 & 7.524 & 10.019 \\
\hline ANTIBIOTICO PARENTERALE & 6.110 & 6.246 & 6.437 & 7.534 & 10.026 \\
\hline Stabilizza & 5.914 & 5.998 & 6.091 & 6.394 & 6.499 \\
\hline NO-Switch & 5.947 & 6.052 & 6.169 & 6.534 & 6.663 \\
\hline Ricovero Breve & 6.054 & 6.054 & 6.054 & 6.224 & 6.236 \\
\hline Ricovero Lungo & 5.920 & 6.052 & 6.198 & 6.612 & 6.770 \\
\hline Switch & 5.864 & 5.916 & 5.975 & 6.183 & 6.253 \\
\hline Ricovero Breve & 5.826 & 5.826 & 5.827 & 5.899 & 5.909 \\
\hline Ricovero Lungo & 5.920 & 6.052 & 6.198 & 6.612 & 6.770 \\
\hline Cambia antibiotico & 6.461 & 6.665 & 6.688 & 8.634 & 10.391 \\
\hline Invio in UTI & 10.057 & 10.634 & 11.272 & 16.616 & 25.266 \\
\hline \multicolumn{6}{|l|}{ Morte precoce } \\
\hline ANTIBIOTICO ORALE & 6.081 & 6.215 & 6.403 & 7.482 & 9.990 \\
\hline Stabilizza & 5.882 & 5.962 & 6.049 & 6.326 & 6.425 \\
\hline Ricovero Breve & 5.826 & 5.826 & 5.827 & 5.899 & 5.909 \\
\hline Ricovero Lungo & 5.920 & 6.052 & 6.198 & 6.612 & 6.770 \\
\hline Cambia antibiotico & 6.461 & 6.665 & 6.688 & 8.634 & 10.391 \\
\hline Invio in UTI & 10.057 & 10.634 & 11.272 & 16.616 & 25.266 \\
\hline \multicolumn{6}{|l|}{ Morte precoce } \\
\hline TERAPIA DOMICILIARE & 2.144 & 2.719 & 3.380 & 4.054 & 7.038 \\
\hline ANTIBIOTICO PARENTERALE & 2.298 & 2.888 & 3.548 & 4.341 & 7.290 \\
\hline Stabilizza & 1.911 & 2.277 & 2.432 & 3.217 & 3.398 \\
\hline NO-Switch & 2.021 & 2.404 & 2.578 & 3.563 & 3.776 \\
\hline Switch & 1.747 & 2.085 & 2.213 & 2.697 & 2.830 \\
\hline Cambia antibiotico & 2.753 & 3.186 & 3.410 & 3.961 & 4.423 \\
\hline Ricovero dopo iniziale insuccesso & 9.316 & 9.665 & 9.297 & 11.426 & 11.499 \\
\hline \multicolumn{6}{|l|}{ Morte precoce } \\
\hline ANTIBIOTICO ORALE & 1.989 & 2.550 & 3.213 & 3.767 & 6.786 \\
\hline Stabilizza & 1.612 & 1.948 & 2.076 & 2.650 & 2.797 \\
\hline Cambia antibiotico & 2.160 & 2.529 & 2.687 & 3.048 & 3.376 \\
\hline Ricovero dopo iniziale insuccesso & 9.166 & 9.512 & 9.151 & 11.087 & 11.158 \\
\hline
\end{tabular}

Tabella 22

Costo medio/paziente guarito a carico della società in funzione del livello di rischio di morte e del percorso terapeutico, tenuto conto della frequenza di ciascun percorso come si realizza in un ipotetico ambito socio-assistenziale, secondo quanto prefigurato dal modello.

Morte precoce 
milioni per UTI. Tuttavia, ridurre la durata dei ricoveri, mentre contribuisce a rendere più efficiente il sistema ospedaliero e ad evitare eccessivi deficit di bilancio, può comportare un certo aumento dei costi dell'assistenza domiciliare e quindi anche degli oneri complessivi a carico del SSN.

Condizioni ottimali di spesa per il SSN, per la società ed anche per l'ospedale si raggiungono riducendo al minimo indispensabile il numero di ricoveri e selezionando i pazienti da ricoverare in base al rischio di morte. Si deve osservare, tuttavia, come il ricovero sia molte volte determinato da fattori sociali e culturali piuttosto che da criteri clinici robusti e validati (12-14). La simulazione di scenari differenti risulta utile per stabilire quale potrebbe essere il risparmio o la perdita correlata a determinate soluzioni e quanto valga la pena cercare di realizzarle a tutti i costi, anche ricorrendo a pressioni o regole vincolanti ma non condivise.

La struttura del modello consente di valutare il costo sostenuto dal paziente e dai familiari, dagli amici o dai volontari, per completare

\begin{tabular}{|c|c|c|c|c|c|}
\hline \multirow{3}{*}{ PERCORSI } & \multicolumn{5}{|c|}{$\begin{array}{l}\text { COSTO MEDIO/PAZIENTE GUARITO } \\
\text { A CARICO DEL SSN (Lire x 1.000) }\end{array}$} \\
\hline & \multicolumn{5}{|c|}{ Rischio di morte (Classe a fine) } \\
\hline & $\mathrm{I}^{\circ}$ & $\mathrm{II}^{\circ}$ & $\mathrm{III}^{\circ}$ & $\mathrm{IV}^{\circ}$ & $\mathrm{V}^{\circ}$ \\
\hline COSTO MEDIO GENERALE & 1.952 & 3.114 & 4221 & 5.148 & 6.833 \\
\hline RICOVERO IN OSPEDALE & 4.764 & 4.782 & 4.835 & 5.395 & 6.841 \\
\hline ANTIBIOTICO PARENTERALE & 4.768 & 4.786 & 4.839 & 5.400 & 6.844 \\
\hline Stabilizza & 4.663 & 4.663 & 4.671 & 4.720 & 4.736 \\
\hline NO-Switch & 4.662 & 4.663 & 4.673 & 4.721 & 4.740 \\
\hline Ricovero Breve & 4.936 & 4.936 & 4.937 & 5.106 & 5.115 \\
\hline Ricovero Lungo & 4.594 & 4.594 & 4.607 & 4.624 & 4.645 \\
\hline Switch & 4.663 & 4.663 & 4.668 & 4.718 & 4.732 \\
\hline Ricovero Breve & 4.708 & 4.709 & 4.709 & 4.780 & 4.789 \\
\hline Ricovero Lungo & 40.594 & 4.594 & 4.607 & 4.624 & 4.645 \\
\hline Cambia antibiotico & 4.708 & 4.764 & 4.680 & $\overline{5.870}$ & 6.835 \\
\hline Invio in UTI & 7.239 & 7.569 & 7.933 & 11.106 & 16.668 \\
\hline \multicolumn{6}{|l|}{ Morte precoce } \\
\hline ANTIBIOTICO ORALE & 4.748 & 4.766 & 4.820 & 5.375 & 6.828 \\
\hline Stabilizza & 40.640 & 4.640 & 4.648 & 4.687 & 4.703 \\
\hline Ricovero Breve & 4.708 & 4.709 & 4.709 & 4.780 & 4.789 \\
\hline Ricovero Lungo & 4.594 & 4.594 & 4.607 & 4.624 & 4.645 \\
\hline Cambia antibiotico & 4.709 & 4.764 & 4.680 & 5.870 & 6.835 \\
\hline Invio in UTI & 7.239 & 7.569 & 7.933 & 11.106 & 16.668 \\
\hline Morte precoce & & & & & \\
\hline
\end{tabular}

\begin{tabular}{|crrrrr}
\hline \hline TERAPIA DOMICILIARE & 1.066 & 1.515 & 2.050 & 2.311 & 4.856 \\
\hline ANTIBIOTICO PARENTERALE & 1.220 & 1.684 & 2.217 & 2.598 & 5.108 \\
\hline Stabilizza & 869 & 1.121 & 1.163 & 1.608 & 1.672 \\
\hline NO-Switch & 979 & 1.249 & 1.309 & 1.954 & 2.051 \\
\hline Switch & 705 & 930 & 945 & 1.088 & 1.104 \\
\hline \hline Cambia antibiotico & 1.598 & 1.918 & 2.028 & 2.351 & 2.580 \\
\hline Ricovero dopo iniziale insuccesso & 7.634 & 7.961 & 7.659 & 8.776 & 8.832 \\
\hline Morte precoce & & & & & \\
\hline ANTIBIOTICO ORALE & 912 & 1.347 & 1.882 & 2.024 & 4.604 \\
\hline Stabilizza & 568 & 793 & 808 & 1.041 & 1.071 \\
\hline Cambia antibiotico & 1.005 & 1.260 & 1.305 & 1.437 & 1.532 \\
\hline Ricovero dopo iniziale insuccesso & 7.483 & 7.809 & 7.512 & 8.437 & 8.491 \\
\hline
\end{tabular}

Tabella 23

Costo medio/paziente guarito a carico della SSN in funzione del livello di rischio di morte e del percorso terapeutico, tenuto conto della frequenza di ciascun percorso come si realizza in un ipotetico ambito socio-assistenziale, secondo quanto prefigurato dal modello. 
Tabella 24

Costo medio/paziente guarito a carico dell'ospedale in funzione del livello di rischio di morte e del percorso terapeutico, tenuto conto della frequenza di ciascun percorso come si realizza in un ipotetico ambito socio-assistenziale, secondo quanto prefigurato dal modello.

\begin{tabular}{|crrrrr}
\hline & \multicolumn{5}{c}{ COSTO MEDIO/PAZIENTE GUARITO } \\
& \multicolumn{5}{c}{ A CARICO DELL'OSPEDALE (Lire x 1.000) } \\
\cline { 2 - 6 } PERCORSI & \multicolumn{5}{c}{ Rischio di morte (Classe a fine) } \\
& $\mathrm{I}^{\circ}$ & \multicolumn{1}{c}{$\mathrm{II}^{\circ}$} & \multicolumn{1}{c}{$\mathrm{III}^{\circ}$} & \multicolumn{1}{c}{$\mathrm{IV}^{\circ}$} & \multicolumn{1}{c}{$\mathrm{V}^{\circ}$} \\
\hline COSTO MEDIO GENERALE & 1.585 & 3.163 & 5.429 & 9.484 & 17.016 \\
\hline RICOVERO IN OSPEDALE & 5.306 & 5.845 & 6.656 & 10.190 & 17.065 \\
\hline ANTIBIOTICO PARENTERALE & 5.329 & 5.871 & 6.684 & 10.227 & 17.090 \\
\hline Stabilizza & 4.362 & 4.715 & 5.070 & 6.138 & 6.506 \\
\hline NO-Switch & 4.881 & 5.323 & 5.768 & 7.103 & 7.562 \\
\hline Ricovero Breve & 2.291 & 2.291 & 2.291 & 2.293 & 2.297 \\
\hline Ricovero Lungo & 5.531 & 6.084 & 6.641 & 8.311 & 8.886 \\
\hline Switch & 3.584 & 3.805 & 4.026 & 4.693 & 4.924 \\
\hline Ricovero Breve & 2.291 & 2.291 & 2.291 & 2.293 & 2.297 \\
\hline Ricovero Lungo & 5.531 & 6.084 & 6.641 & 8.311 & 8.886 \\
\hline Cambia antibiotico & 7.319 & 7.946 & 8.393 & 11.557 & 14.878 \\
\hline Invio in UTI & 24.595 & 26.216 & 28.006 & 49.261 & 80.227 \\
\hline Morte precoce & & & & & \\
\hline ANTIBIOTICO ORALE & 5.212 & 5.741 & 6.543 & 10.041 & 16.963 \\
\hline Stabilizza & 4.232 & 4.563 & 4.896 & 5.897 & 6.242 \\
\hline Ricovero Breve & 2.291 & 2.291 & 2.291 & 2.293 & 2.297 \\
\hline Ricovero Lungo & 5.531 & 6.084 & 6.641 & 8.311 & 8.886 \\
\hline Cambia antibiotico & 7.319 & 7.946 & 8.393 & 11.557 & 14.878 \\
\hline Invio in UTI & 24.595 & 26.216 & 28.006 & 49.261 & 80.227 \\
\hline Morte precoce & & & & & \\
\hline \hline & & & & &
\end{tabular}

\section{Tabella 25}

Confronto tra situazione base e ipotetica applicazione integrale dell'indicazione di non ricoverare i pazienti a basso rischio di morte (classe di Fine 1-3). I valori indicati rappresentano il costo medio per paziente trattato, tenuto conto delle frequenze di realizzazione dei diversi percorsi.

\begin{tabular}{|c|c|c|c|c|c|c|c|c|c|}
\hline \multirow{3}{*}{ Stratificazioni } & \multicolumn{9}{|c|}{ COSTO MEDIO/PAZIENTE TRATTATO (Lire x 1.000) } \\
\hline & \multicolumn{3}{|c|}{ SOCIETA' } & \multicolumn{3}{|c|}{ SSN } & \multicolumn{3}{|c|}{ OSPEDALE } \\
\hline & BASE & IDEALE & Diff. & BASE & IDEALE & Diff & BASE & IDEALE & Diff \\
\hline $\begin{array}{l}\text { TUTTI I } \\
\text { PAZIENTI }\end{array}$ & 4.870 & 3.919 & 951 & 3.368 & 2.485 & 883 & 988 & 3.594 & 1.394 \\
\hline $\mathrm{FINE} \mathrm{I}^{\circ}$ & 09 & 2.129 & 940 & 37 & 1.059 & 878 & .573 & 409 & 1.164 \\
\hline Ospedale & 6.048 & ----- & 6.048 & 4.720 & ----- & 4.720 & 5.257 & ----- & 5.257 \\
\hline Domicilio & 2.129 & 2.129 & 0 & 1.059 & 1.059 & 0 & 409 & 409 & 0 \\
\hline FINE II $^{\circ}$ & 4.389 & 2.690 & 1.699 & 3.076 & 1.499 & 1.577 & 3.125 & 587 & 2.538 \\
\hline Ospedale & 6.157 & ---- & 6.157 & 4.718 & ----- & 4.718 & 5.767 & ---- & 5.767 \\
\hline Domicilio & 2.690 & 2.690 & 0 & 1.499 & 1.499 & 0 & 587 & 587 & 0 \\
\hline FINE III $^{\circ}$ & 5.665 & 3.334 & 2.331 & 4.153 & 2.022 & 2.131 & 5.341 & 1.074 & 4.267 \\
\hline Ospedale & 6.323 & ----- & 6.323 & 4.754 & ----- & 4.754 & 6.545 & ---- & 6.545 \\
\hline Domicilio & 3.334 & 3.334 & 0 & 2.022 & 2.022 & 0 & 1.074 & 1.074 & 0 \\
\hline 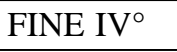 & 6.561 & 6.811 & -250 & 4.661 & 4.884 & -223 & 8.588 & 9.226 & -638 \\
\hline Ospedale & 6.811 & 6.811 & 0 & 4.884 & 4.884 & 0 & 9.226 & 9.226 & 0 \\
\hline Domicilio & 3.682 & ---- & 3.682 & 2.099 & ----- & 2.099 & 1.263 & ----- & 1.263 \\
\hline FINE $V^{\circ}$ & 7.251 & 7.259 & -8 & 4.951 & 4.957 & -6 & 12.329 & 12.365 & -36 \\
\hline Ospedale & 7.259 & 7.259 & 0 & 4.957 & 4.957 & 0 & 12.365 & 12.365 & 0 \\
\hline Domicilio & 5.124 & ---- & 5.124 & 3.536 & $-\cdots$ & 3.536 & 3.555 & ---- & 3.555 \\
\hline
\end{tabular}


un ciclo di trattamento della CAP. Alcune voci di costo a carico del paziente, che in genere sono trascurate, sono risultate sensibili nel determinare il valore del costo sociale. La perdita di produttività è risultato il secondo fattore sensibile, preceduto solo dal valore della tariffa DRG..

In questo lavoro non abbiamo volutamente confrontato due o più antibiotici o protocolli di terapia antibiotica, ma solo in generale il trattamento parenterale e quello orale. La struttura del modello può, tuttavia, essere utilizzata per attuare una completa analisi costo/efficacia di confronto fra due o più antibiotici.
In conclusione, l'albero delle decisioni messo a punto per analizzare la gestione del paziente affetto da CAP è risultato flessibile ed adattabile alla maggior parte delle situazioni prevedibili. La sua struttura è forse eccessivamente complessa, ma presenta il vantaggio di rappresentare tutte le opzioni che praticamente si possono presentare nel percorso clinicoterapeutico della polmonite dell'adulto.

La validità del modello e la sua utilità applicativa potrà essere definitivamente confermata allorquando si renderanno disponibili dati clinici ed epidemiologici sulla CAP raccolti direttamente sul campo.

\section{BIBLIOGRAFIA}

1. Eandi M.: Percorsi diagnostico-terapeutici nella gestione dei pazienti affetti da polmonite acquisita in comunità. Farmeconomia e Percorsi Terapeutici 2000; 1: 35-52

2. De Lalla F, Eandi M.: Indagine sull'uso di antibiotici nel trattamento delle infezioni delle basse vie respiratorie negli ospedali italiani: considerazioni farmacoeconomiche. Giornale Italiano di Chemioterapia 1998; 45: 59-87.

3. Dans PE, Charache P, Fahey M, Otter SE.: Management of pneumonia in the prospective payment era: a need for more clinician and support service interaction. Arch Intern Med 1984; 144: 1392-1397.

4. Garibaldi RA.: Epidemiology od community-acquired respiratory tract infections in adults; incidence, etiology, and impact. Am J Med 1985; 78: 32-37.

5. La Force FM.: Community-acquired lower respiratory tract infections: prevention and cost-control strategies. Am J Med 1985; 78: 52-57.

6. Lave JR, Fine MJ, Sankey SS, Hanusa BH, Weissfeld LA, Kapoor WN.: Hospitalized pneumonia: outcomes, treatment patterns, and costs in urban and rural areas. J Gen Intern Med 1996; 11: 415-421.

7. Fine MJ, Hough LJ, Medsger AT, Li YH, Ricci EM, Singer DE, Marrie TJ, Coley CM, Walsh MB, Karpf M, Lahive KC, Kapoor N.: Hospital admission decision for patients with community-acquired pneumonia: results from the Penumonia PORT cohort study. Arch Intern Med 1997a; 157: 36-44.

8. Eandi M, Bondonio PV, Dirindin N.: Farmacoeconomia e Terapia Antibiotica. ABE, Milano, 1993.

9. Weinstein MC, Fineberg HV.: Clinical Decision Analysis. Philadelphia: W.B. Saunders, 1980.

10. Fine MJ, Smith MA, Carson CA, et al.: Prognosis and outcomes of patients with community-acquired pneumonia: a meta-analysis. JAMA 1996; 275: 134-141.

11. Fine MJ, Auble TE, Yealy DM, Hanusa BH, Weissfeld LA, Singer DE, Coley CM, Marrie TJ, Kapoor WN.: $A$ prediction rule to identify low-risk patients with community-acquired pneumonia. NEngl J Med 1997; 336: $243-250$.

12. Schaberg T, Gialdroni-Grassi G, Huchon G, Leophonte P, Manresa F, Woodhead M.: An analysis of decisions by European general practitioners to admit to hospital patients with lower respiratory tract infections. Thorax 1996; 51: 1017-1022.

13. Woodhead M, Huchon G, Gialdroni-Grassi G, Leophonte P, Manresa F, Schaberg T.: Investigation of lower respiratory tract infection in the community: a European survey. Eur Respir J 1996;

14. Huchon GJ, Gialdroni-Grassi G, Leophonte P, Manresa F, Schaberg T, Woodhead M.: Initial antibiotic therapy for lower respiratory tract infection in the community : a European survey. Eur Respir J 1996; 9: 1590-1595. 